\title{
Effective properties of periodic fibrous electro-elastic composites with mechanic imperfect contact condition
}

\author{
R. Rodríguez-Ramos ${ }^{\mathrm{a},}{ }^{*}$, R. Guinovart-Díaz ${ }^{\mathrm{a}}$, J.C López-Realpozo $^{\mathrm{a}}$, J. Bravo-Castillero ${ }^{\mathrm{a}}$,J.A. Otero $^{\mathrm{b}}$, F.J. Sabina ${ }^{\mathrm{c}}$, F. Lebon ${ }^{\mathrm{d}}$ \\ aFacultad de Matemática y Computación, Universidad de La Habana, San Lázaro y L Vedado Habana 4, CP 10400, Cuba \\ 'Instituto de Cibernética, Matemática y Física, ICIMAF. Calle 15 No. 551, entre C y D. Vedado, Habana 4, CP 10400, Cuba \\ 'Instituto de Investigaciones en Matemáticas Aplicadas y en Sistemas Universidad Nacional Autónoma de México, Apartado Postal 20-726 Delegación de \\ Álvaro Obregón, 01000 México, DF, México \\ dLaboratoire de Mécanique et d'Acoustique, Université Aix-Marseille, CNRS, Centrale Marseille, 31 Chemin Joseph-Aiguier, 13402 Marseille Cedex 20, France
}

\begin{abstract}
In this work, two-phase parallel fiber-reinforced periodic piezoelectric composites are considered wherein the constituents exhibit transverse isotropy and the cells have different configurations. Mechanical imperfect contact at the interface of the composites is studied via linear spring model. The statement of the problem for two phase piezoelectric composites with mechanical imperfect contact is given. The local problems are formulated by means of the asymptotic homogenization method (AHM) and their solutions are found using complex variable theory. Analytical formulae are obtained for the effective properties of the composites with spring imperfect type of contact and different parallelogram cells. Some numerical examples and comparisons with other theoretical results illustrate that the model is efficient for the analysis of composites with presence of parallelogram cells and the aforementioned imperfect contact.
\end{abstract}

\section{Introduction}

Nowadays, piezoelectric materials have a key role in manufacturing of sensors and actuators, which may be used for active control of elastic deformations and vibrations of the structures. These materials have a wide range of applications in science and technology such as in ultrasonic transducers, sonar projects, and under water acoustic.

In order to successfully integrate piezoelectric actuators into structures, the physical nature of the interface condition between the actuators and the base structure, and its effect on the induced electro-mechanical field must be fully understood.

Some years ago, Hashin [1] using the generalized self-consistent scheme studied the thermoelastic properties of unidirectional fiber composites with imperfect interface conditions defined in terms of linear relations between interface tractions and displacement jumps. Besides, the asymptotic scheme for the analysis of dilute elastic composites, which includes circular inclusions with imperfect bonding at the interface, is presented by Bigoni et al. [2]. The interface, in this work, is characterized by a discontinuous displacement field across it, linearly related to the tractions. Recently, an

\footnotetext{
* Corresponding author. Tel.: +537832 2466 .

E-mail address: reinaldo@matcom.uh.cu (R. Rodríguez-Ramos).
}

asymptotic approach for simulation of the imperfect interfacial bonding in composite materials is proposed by Andrianov et al. [3] where a problem of the axial shear of elastic fibre-reinforced composites with square and hexagonal arrays of cylindrical inclusions is considered. The performed analysis is based on the asymptotic homogenization method and the cell problem is solved using the underlying principles of the boundary shape perturbation technique. Moreover, the effective elastic moduli of composite materials are investigated by Yanase and Ju [4] in the presence of imperfect interfaces between the inclusions and the matrix. The primary focus is on the spherical particle reinforced composites. By admitting the displacement jumps at the particle-matrix interface, the modified Eshelby inclusion problem is studied. Besides, Chen et al. [5] studied a micromechanical method based on generalized method of cells for investigating elastic and plastic response of composites subjected to off-axis loading. To date, to the best of the author's knowledge, the problems associated with piezoelectric materials and inhomogeneities with imperfect interface conditions have not been reported intensively in the literature. For instance, piezothermoelastic constitutive laws at a weak interface is analyzed by Shu [6]; Shodja et al. [7] examine the electro-mechanical fields for a circular anisotropic piezoelectric fiber sensor inside an anisotropic piezoelectric or non-piezoelectric elastic matrix with imperfect interface under remote in-plane uniform tension, among other works. 
Different authors are investigating the behavior of composites with non-perfect bonding contact. Recently, an asymptotic study of different types of imperfect interfaces arising in the problem of conduction through a granular composite material was presented in [8]. In Andrianov et al. [3], imperfection is considered by means of a discontinuity of the displacement (spring model). However, it may look natural that the mechanical weakening of the interface (due to delamination, decohesion, etc.) should also induce the decrease of the electric contact. For instance, in piezoelectric material, due to their electro-mechanical coupling, there exist induced electric charges when a mechanical loading is applied [9]. Although the electro-mechanical coupling exists, the mechanical contribution is remarkable in the behavior of the composites. In this sense, as a first approximation in the study of piezoelectric composites under imperfect contact (mechanic and electric) we assume only mechanical imperfect adherence.

The present work is motivated by the interest to study the influence of imperfect contact over the effective piezoelectric response when the composites have oblique fibrous orientation. Composites with rhombus periodic cell are important since they could describe monoclinic behavior of certain physical and biological structures. This is an extension of previous results reported by Bravo-Castillero et al. [10] and Sabina et al. [11] where perfect contact for piezoelectric composites was considered. Moreover, in this contribution other recent researches related to composites with perfect contact conditions and parallelogram cells studied by Guinovart-Díaz et al. [12,13] and Rodríguez-Ramos et al. [14] are extended to composites with the same distribution of the periodic cells but now with no-well bonding contact. The interface imperfection is posed on the mechanical fields only. The mechanical behavior of imperfect interface is modeled via an idealization of a layer of mechanical springs of zero thickness. The vanishing value of $\tilde{K}_{n}$ and $\tilde{K}_{t}, \tilde{K}_{s}$ corresponds to pure debonding (normal perfect debonding), in-plane pure sliding, and out-of-plane pure sliding, respectively. The status of the mechanical bonding is completely determined by appropriate values of these constants. For large enough values of the constants, the perfect bonding interface is achieved. The spring approach is used for the calculation of the piezoelectric effective coefficients in a composite with different angular distribution of fibers. Using the two scale asymptotic homogenization method the formulation of the local problems for linear two phase piezoelectric composites with parallelogram cell and mechanical imperfect contact conditions is given and the solution of each plane local problems is found using the potential methods of a complex variable and the properties of doubly periodic Weierstrass elliptic functions. Besides, the complete set of analytical expressions for the piezoelectric coefficients of a fiber reinforced composite with circular cylindrical shape periodically distributed in the matrix under linear spring imperfect contact conditions are obtained via AHM. The study of such composites with mechanic and electric coupled effect is an extension of previous works considered by Molkov and Pobedria [15], Rodríguez-Ramos et al. [16] and Lopez-Realpozo et al. [17] where only the elastic properties of the composite with mechanical imperfect contact were analyzed. In particular, the last two works are referred only to antiplane elastic properties.

The heterogeneous problem formulation is presented in Section 1 where the basic equations and the general statement of the imperfect conditions are written. In Section 2 the two scale asymptotic homogenization algorithm is developed and the statement of the plane and antiplane local problems with mechanical (spring) imperfect conditions are written. Solutions of each local problem are given in Sections 3 and 4. Moreover, Section 5 is devoted to present some important parameters used for evaluating the performance of 1-3 piezoelectric composites. In Section 6 validations of the present model and comparisons with other theoretical and experimental approaches are shown as well as the effect of the imperfect adherence in the ultrasonic transducers applications. Finally, some conclusions are written.

\section{Heterogeneous problem formulation}

Consider piezoelectric materials that respond linearly to changes in the mechanic and electric fields. A two-phase uniaxial reinforced material is considered here in which fibers and matrix have homogeneous and transversely isotropic properties; the axis of transverse symmetry coincides with the fiber direction, which is taken as the $\mathrm{Ox}_{3}$-axis. The fiber cross-section is circular. Moreover, the fibers are periodically distributed without overlapping in directions parallel to the $O w_{1}$ - and $O w_{2}$-axis, where $w_{1} \neq 0$ and $w_{2} \neq 0\left(w_{2} \neq \lambda w_{1}, \lambda \in \mathbb{R}\right)$ are two complex numbers which define the parallelogram periodic cell of the two-phase composite. Therefore the composite $\Omega$ consists of a parallelogram array of identical circular cylinders embedded in a homogeneous medium (Fig. 1). The cylinders are infinitely long.

The response of the material at the microscale level is analyzed using representative material elements (RME) or Representative Cell. The fiber-reinforced material is assumed to have a periodic arrangement of fine scale fibers embedded in a matrix. A sample RME is shown in Fig. 2 where the appropriate periodic unit cell $Y$ is taken as a regular parallelogram in the $y_{1} y_{2}$-plane so that $Y=Y_{1} \cup Y_{2}$ with $Y_{1} \cap Y_{2}=\phi$, the domain $Y_{1}$ is occupied by the matrix and its complement $Y_{2}$ a circle of radius $R$, is filled up with the fiber for a piezoelectric composite with rectangle, rhombic and parallelogram arrangements of unidirectional fibers. A local Cartesian coordinate system $\mathbf{y}$ is introduced at the microscale and oriented such that the $y_{3}$-axis is aligned parallel to the axis of the fibers. The microscale coordinates $\mathbf{y}$ of a point in the RME are related to the macroscale coordinates $\mathbf{x}$ by $\mathbf{y}=\mathbf{x} / \varepsilon$, where $\varepsilon \ll 1$. Beside the use of subscript, matrix and fiber associated quantities are also referred below by means of superscripts in brackets (1) and (2), respectively. Twophase composite is considered which comprises a matrix with homogeneous properties given by the following moduli tensors: elastic $C_{i j k l}^{(1)}$, piezoelectric $e_{i j k}^{(1)}$ and dielectric permittivity $\kappa_{i j}^{(1)}$, in which are embedded parallel circular cylindrical fibers with corresponding homogeneous properties $C_{i j k l}^{(2)}, e_{i j k}^{(2)}$ and $\kappa_{i j}^{(2)}$.

To denote the dependence of a field variable on the macroscale and microscale coordinates, the superscript $\varepsilon$ is used, i.e. $\mathscr{F}^{\varepsilon}=\mathscr{F}(\mathbf{x}, \mathbf{y})$ where $\mathscr{F}^{\varepsilon}$ represents a scalar, vector or tensor field. All field variables are assumed to depend on the coordinates of both scales. For an arbitrary microstructure, material phases, and therefore material properties such as elastic constants, are

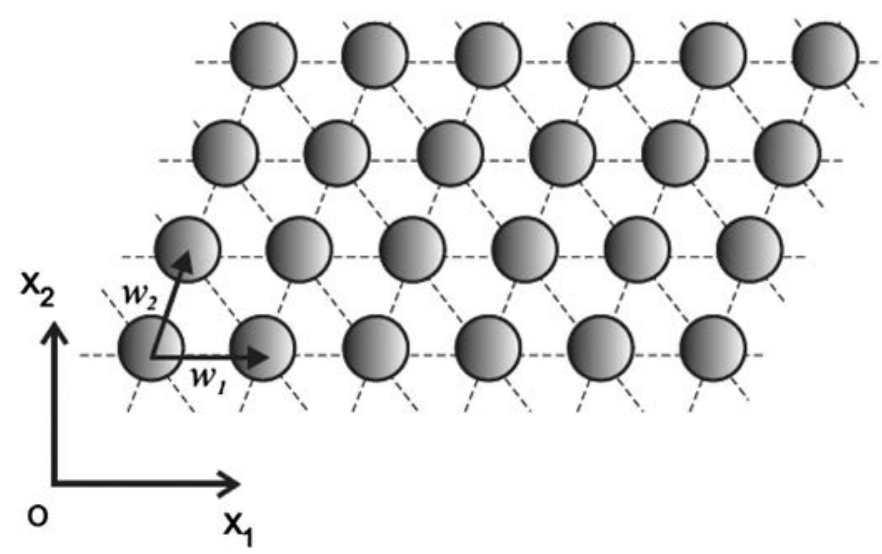

Fig. 1. The heterogeneous medium and extracted the parallelogram periodic cell. 

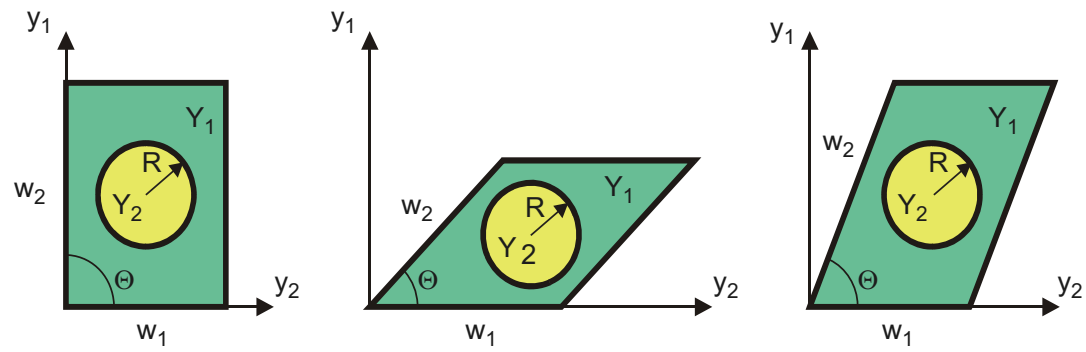

Fig. 2. Different unit cells - rectangle, rhombic and parallelogram.

functions of the microscale coordinates $\mathbf{y}$. In the following formulation, indicial and direct notation will be used interchangeably.

The local governing mechanical and charge equilibrium equations in the absence of body forces and free charges are

$\sigma_{i j, j}^{\varepsilon}=0, D_{i, i}^{\varepsilon}=0$ in $\Omega$

where the subscript comma denotes partial differentiation, $\sigma_{i j}^{\varepsilon}$ are the components of the Cauchy stress tensor and $D_{i}^{\varepsilon}$ are the components of the electric displacement vector.

Using the conventional indicial notation in which repeated subscripts are summed over the range of $i, j, k, l=1,2,3$, the constitutive equations are

$\sigma_{i j}^{\varepsilon}=C_{i j k l} \varepsilon_{k l}^{\varepsilon}-e_{k i j} E_{k}^{\varepsilon}, D_{i}^{\varepsilon}=e_{i k l} \varepsilon_{k l}^{\varepsilon}+\kappa_{i k} E_{k}^{\varepsilon}$,

where $\varepsilon_{k l}^{\varepsilon}$ is the infinitesimal strain tensor and $E_{k}^{\varepsilon}$, is the electric field vector. The quantities $C_{i j k l}, e_{k i j}, \kappa_{i k}$ are components of the elastic stiffness tensor, the piezoelectric tensor, and the dielectric permittivity tensor, respectively.

The gradient equations, which involve the strain-displacement equations and electric field-potential, are

$\varepsilon_{k l}^{\varepsilon}=\frac{1}{2}\left(\frac{\partial u_{k}^{\varepsilon}}{\partial x_{l}}+\frac{\partial u_{l}^{\varepsilon}}{\partial x_{k}}\right), E_{k}^{\varepsilon}=-\phi_{, k}^{\varepsilon}$,

where $u_{k}^{\varepsilon}$ and $\phi^{\varepsilon}$ are the mechanical displacement and the electric potential, respectively.

The material constants are assumed to satisfy the symmetries $C_{i j k l}=C_{j i k l}=C_{k l i j}, e_{k i j}=e_{k j i}, \kappa_{i k}=\kappa_{k i}$. In addition, the elasticity tensor and the dielectric permittivity tensor are assumed to be positive definite.

Substituting (2) and (3) into (1) we obtain a coupled system of partial differential equations with coefficients rapidly oscillating

$\left(C_{i j k l}(\mathbf{y}) u_{k, l}^{\varepsilon}+e_{k i j}(\mathbf{y}) \phi_{, k}^{\varepsilon}\right)_{, j}=0, \quad\left(e_{i k l}(\mathbf{y}) u_{k, l}^{\varepsilon}-\kappa_{i k}(\mathbf{y}) \phi_{, k}^{\varepsilon}\right)_{, i}=0$ in $\Omega$.

Eq. (4) represents a system of equations for finding $u_{i}$ and $\phi$. For a complete solution, it is necessary to assign suitable boundary conditions, for instance

$u_{i}^{\varepsilon}=\hat{u}_{i} ; \quad \sigma_{i j}^{\varepsilon} n_{j}=\hat{S}_{i} ; \quad \phi^{\varepsilon}=\phi_{0} ; \quad D_{i}^{\varepsilon} n_{i}=0$ on $\partial \Omega$,

where $\hat{u}_{i}, \hat{S}_{i}$ and $\phi_{0}$ are the prescribed displacement, force and electric potential on the boundary of the composite, respectively.

The interface conditions are specified as follows. The inclusion problems associated with piezocomposite materials, which have been presented in the literature, are mainly concerned with perfect interface condition; see for example, the works of Avellaneda and Swart [18] and Gibiansky and Torquato [19]. In the case of perfect bonding, the continuities of displacement, traction, electric potential, and normal electric displacement are concerned. Often, the above electro-mechanical interface conditions are not realistic assumptions in modeling the actual physical problems. In this section it is intended to analyze the behavior of a piezocomposite under only mechanical imperfect contact. The mechanical behavior of imperfect interface is modeled via an idealization of a layer of mechanical springs of zero thickness. The spring constants are the measures for the magnitude of the associated continuities. The vanishing value of $\tilde{K}_{n}$ and $\tilde{K}_{t}, \tilde{K}_{s}$ corresponds to pure debonding (normal perfect debonding), in-plane pure sliding, and out-of-plane pure sliding, respectively. The status of the mechanical bonding is completely determined by appropriate values of these constants. For large enough values of the constants, the perfect bonding interface is achieved.

The spring stiffness matrix, the mechanic displacement and the traction vectors using the vector notation are written as

$\mathbf{u}=\left(\begin{array}{l}u_{n} \\ u_{t} \\ u_{s}\end{array}\right), \mathbf{T}=\left(\begin{array}{c}T_{n} \\ T_{t} \\ T_{s}\end{array}\right), \mathbf{K}=\left(\begin{array}{ccc}\tilde{K}_{n} & 0 & 0 \\ 0 & \tilde{K}_{t} & 0 \\ 0 & 0 & \tilde{K}_{s}\end{array}\right)$

The effect of mechanical imperfection is incorporated through the mechanical displacements jumps across the interface, while the corresponding tractions, electric potential and normal electric displacement remain continuous. Several examples addressing the effect of electro-mechanical imperfections on the induced electromechanical fields are thoroughly examined by Shodja et al. [9]. There are seven types of imperfections considered in Table 1 of the work of Shodja et al. [9]. Mechanical partial debonding (type of imperfection VII) listed in Table 1 is focused in the present work. Hence, the mechanical imperfect condition considered in Shodja et al. [7] and Hashin [1] may be expressed as

$\mathbf{T}^{(1)}+\mathbf{T}^{(2)}=0, \mathbf{T}^{(\gamma)}=(-1)^{\gamma+1} \mathbf{K}[\mathbf{u}],[\phi]=0,[\mathbf{D}] \mathbf{n}=0$ on $\Gamma$.

In these relations [•] indicates the jump in the quantity at the common interface $\Gamma$ between the fiber and the matrix; $\mathbf{n}$ is the outward unit normal on $\Gamma ; u_{n}, u_{t}, u_{s}$ are the normal and the two tangential components of the mechanic displacement vector, respectively; $T_{n}, T_{t}, T_{s}$ are the normal and tangential components of the traction vector $\mathbf{T}\left(T_{i}=\sigma_{i j} n_{j}\right)$. The superscripts $(\gamma), \gamma=1,2$ denote the matrix and fiber respectively.

In order to study the imperfect contact conditions, the relations between the displacement and traction vectors (6) are related to their Cartesian representations by the following expressions:

$\left(\begin{array}{l}u_{n} \\ u_{t} \\ u_{s}\end{array}\right)=\left(\begin{array}{ccc}\cos \varphi & \sin \varphi & 0 \\ -\sin \varphi & \cos \varphi & 0 \\ 0 & 0 & 1\end{array}\right)\left(\begin{array}{l}u_{1} \\ u_{2} \\ u_{3}\end{array}\right),\left(\begin{array}{l}T_{n} \\ T_{t} \\ T_{s}\end{array}\right)=\left(\begin{array}{ccc}\cos \varphi & \sin \varphi & 0 \\ -\sin \varphi & \cos \varphi & 0 \\ 0 & 0 & 1\end{array}\right)\left(\begin{array}{l}T_{1} \\ T_{2} \\ T_{3}\end{array}\right)$.

Thus, the expression (7) on $\Gamma$, can be rewritten in the following indicial form:

$\mathbf{T}^{(1)}+\mathbf{T}^{(2)}=0$ on $\Gamma, \quad T_{n}^{(\gamma)}=(-1)^{\gamma+1} \tilde{K}_{n}\left[u_{n}\right], \quad T_{t}^{(\gamma)}=(-1)^{\gamma+1} \tilde{K}_{t}\left[u_{t}\right]$,

$T_{s}^{(\gamma)}=(-1)^{\gamma+1} \tilde{K}_{s}\left[u_{s}\right]$ on $\Gamma, \quad[\phi]=0, \quad[\mathbf{D}] \mathbf{n}=0$ on $\Gamma$.

\section{Two scales asymptotic formulation}

The overall properties of the above periodic medium are sought by means of the AHM in the same way as it is developed by Bakhvalov and Panasenko [20] and Bensoussan et al. [21]. In this 
section, the application of the general AHM method using the two scale formulation for the electroelastic heterogeneous problem is presented. As discussed earlier, field variables are assumed to depend on both the macroscale coordinates $\mathbf{x}$ and microscale coordinates $\mathbf{y}$, and therefore, spatial derivatives of the field variables are obtained by use of the chain rule and the relation $\mathbf{y}=\mathbf{x} / \varepsilon$ as

$\frac{\partial \mathscr{F}^{\varepsilon}}{\partial x_{i}}=\frac{\partial \mathscr{F}(\mathbf{x}, \mathbf{y})}{\partial x_{i}}+\frac{1}{\varepsilon} \frac{\partial \mathscr{F}(\mathbf{x}, \mathbf{y})}{\partial y_{i}}$

The AHM method assumes a two-scale asymptotic expansion for the mechanical displacement and electric potential analogous to Bravo-Castillero et al. [10] and Sabina et al. [11]:

$\mathbf{u}^{\varepsilon}(\mathbf{x})=\mathbf{u}^{0}(\mathbf{x}, \mathbf{y})+\varepsilon \mathbf{u}^{1}(\mathbf{x}, \mathbf{y})+\cdots$,

$\phi^{\varepsilon}(\mathbf{x})=\phi^{0}(\mathbf{x}, \mathbf{y})+\varepsilon \phi^{1}(\mathbf{x}, \mathbf{y})+\cdots$,

where the superscripts on the field variables denote the different orders in the asymptotic expansion and do not imply exponentiation. Although $\mathbf{u}^{\varepsilon}$ and $\phi^{\varepsilon}$ depend on coordinates $\mathbf{x}$ and $\mathbf{y}$, it has been previously demonstrated that the lowest order terms $\mathbf{u}^{0}$ and $\phi^{0}$ depend only on the macroscale coordinates $\mathbf{x}$ and correspond to the average macroscale values as $\varepsilon \rightarrow 0$. In other words,

$\lim _{\varepsilon \rightarrow 0} \mathbf{u}^{\varepsilon}=\mathbf{u}^{0}(\mathbf{x}, \mathbf{y})=\overline{\mathbf{u}}(\mathbf{x}), \quad \lim _{\varepsilon \rightarrow 0} \phi^{\varepsilon}=\phi^{0}(\mathbf{x}, \mathbf{y})=\bar{\phi}(\mathbf{x})$,

where $\overline{\mathbf{u}}(\mathbf{x})$ and $\bar{\phi}(\mathbf{x})$ denote the average macroscale displacement and electric potential, respectively. The average is considered in the sense that any field variable $\mathscr{F}^{\varepsilon}$, which is a function of both the macroscale and microscale coordinates, is integrated over the cell domain to obtain the corresponding averaged field variable $\overline{\mathscr{F}}$ which is dependent only on the macroscale coordinates, i.e. $\overline{\mathscr{F}}=(1 /|Y|) \int_{Y} \mathscr{F}^{\varepsilon} d Y$.

As such, the first order terms $\varepsilon \mathbf{u}^{1}(\mathbf{x}, \mathbf{y})$ and $\varepsilon \phi^{1}(\mathbf{x}, \mathbf{y})$ represent the microscale fluctuations in the mechanical displacement and electric potential, respectively. The infinitesimal strains and electric field, which are obtained from (3) and (11) using (10), are substituted into the constitutive equation (2) to obtain the following expansions for the stress and electric displacement:

$\sigma_{i j}^{\varepsilon}=\sigma_{i j}^{0}(\mathbf{x}, \mathbf{y})+O(\varepsilon), \quad D_{i}^{\varepsilon}=D_{i}^{0}(\mathbf{x}, \mathbf{y})+O(\varepsilon)$,

where the zero order terms for the stress and electric displacement are

$\left.\left.\sigma_{i j}^{0}(\mathbf{x}, \mathbf{y})=C_{i j k l} \frac{\partial \bar{u}_{k}}{\partial x_{l}}+\frac{\partial u_{k}^{1}}{\partial y_{l}}\right)+e_{k i j} \frac{\partial \bar{\phi}}{\partial x_{k}}+\frac{\partial \phi^{1}}{\partial y_{k}}\right)$,

$\left.\left.D_{i}^{0}(\mathbf{x}, \mathbf{y})=e_{i k l} \frac{\partial \bar{u}_{k}}{\partial x_{l}}+\frac{\partial u_{k}^{1}}{\partial y_{l}}\right)-\kappa_{i k} \frac{\partial \bar{\phi}}{\partial x_{k}}+\frac{\partial \phi^{1}}{\partial y_{k}}\right)$

Since $\varepsilon \ll 1$, the microscale stress and electric displacement correspond to the first order term in (12). Inserting (12) into the mechanical and charge equilibrium equation (1), multiplying by $\varepsilon$, and taking the limit as $\varepsilon \rightarrow 0$ yields the following local equilibrium equations:

$\frac{\partial \sigma_{i j}^{0}}{\partial y_{j}}=0, \quad \frac{\partial D_{i}^{0}}{\partial y_{i}}=0$.

To proceed with the asymptotic formulation, due to the linearity of this problem and assuming regularity of both the inclusions shapes and the smoothness of the coefficients, the following decompositions are used for $\mathbf{u}^{1}$ and $\phi^{1}$ :

$\mathbf{u}^{1}(\mathbf{x}, \mathbf{y})={ }_{p q} \mathbf{M}(\mathbf{y}) \frac{\partial u_{p}^{0}}{\partial x_{q}}(\mathbf{x})+{ }_{p} \mathbf{P}(\mathbf{y}) \frac{\partial \phi^{0}}{\partial x_{q}}(\mathbf{x})$,

$\phi^{1}(\mathbf{x}, \mathbf{y})={ }_{p q} N(\mathbf{y}) \frac{\partial u_{p}^{0}}{\partial x_{q}}(\mathbf{x})+{ }_{p} Q(\mathbf{y}) \frac{\partial \phi^{0}}{\partial x_{q}}(\mathbf{x})$

where the sets of $p q$-functions, ${ }_{p q} \mathbf{M}(\mathbf{y})$ and ${ }_{p q} N(\mathbf{y})$, and $p$-functions,
${ }_{p} \mathbf{P}(\mathbf{y})$ and ${ }_{p} Q(\mathbf{y})$, depend only on $\mathbf{y}$.They are microscale characteristic functions that relate the macroscale strain and electric field to the microscale fluctuations in the mechanical displacement and electric potential. They are the unique periodic solution of the so-

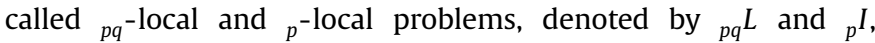
respectively, over the periodic unit cell $Y$, defined below.

The ${ }_{p q} L$ problem seeks displacements ${ }_{p q} \mathbf{M}_{(\gamma)}(\mathbf{y})$ and potential ${ }_{p q} N_{(\gamma)}(\mathbf{y})$, in $Y_{\gamma}, \gamma=1,2$, which are periodic functions of periods $w_{1}=1, w_{2}=b e^{i \Theta}, b>0$ is the modulus of this complex number and are the solution of the following equations

${ }_{p q} \sigma_{i \delta, \delta}^{(\gamma)}=0$ in $Y_{\gamma}$,

${ }_{p q} D_{\delta, \delta}^{(\gamma)}=0$ in $Y_{\gamma}$,

under imperfect mechanical and perfect electrical conditions

$\left.{ }_{p q} \mathbf{T}^{(1)}+{ }_{p q} \mathbf{T}^{(2)}=0, \quad{ }_{p q} N\right]=0 \quad$ on $\Gamma, \quad{ }_{p q} T_{n}^{(\gamma)}=(-1)^{\gamma+1} \tilde{K}_{n}\left[{ }_{p q} M_{n}\right]$,

${ }_{p q} T_{t}^{(\gamma)}=(-1)^{\gamma+1} \tilde{K}_{t}\left[p q \mathrm{M}_{t}\right], \quad{ }_{p q} T_{s}^{(\gamma)}=(-1)^{\gamma+1} \tilde{K}_{s}\left[p q \mathrm{M}_{s}\right]$ on $\Gamma$,

$\left[{ }_{p q} D_{\delta}\right] n_{\delta}=-\left[e_{\delta p q}^{(\gamma)}\right] \mathrm{n}_{\delta}$ on $\Gamma$.

To assure the solution of the ${ }_{p q} L$ problems is unique, the functions also satisfy condition that $\left.{ }_{p q} \mathbf{M}\right\rangle=\left\langle{ }_{p q} N\right\rangle=0$, where the angular brackets define the volume average per unit length over the unit periodic cell $\langle F\rangle=1 /|Y| \int_{Y} F(\mathbf{y}) d \mathbf{y}$.

Moreover,

${ }_{p q} \sigma_{i \delta}^{(\gamma)}=C_{i \delta k \lambda}^{(\gamma)} p q M_{k, \lambda}^{(\gamma)}+e_{\lambda i \delta}^{(\gamma)} p q_{, \lambda} N_{, \lambda}^{(\gamma)}$

${ }_{p q} D_{\delta}^{(\gamma)}=e_{\delta k \lambda}^{(\gamma)}{ }_{p q} M_{k, \lambda}^{(\gamma)}-\kappa_{\delta \lambda}^{(\gamma)}{ }_{p q} N_{, \lambda}^{(\gamma)}$,

the comma notation denotes a partial derivative relative to the $y_{\delta}$ component, i.e. $U_{, \delta} \equiv \partial U / \partial y_{\delta}$; the summation convention is also understood for Greek indices, which run from 1 to 2; no summation is carried out over upper case indices, whether Latin on Greek. The functions ${ }_{p q} M_{t},{ }_{p q} M_{s},{ }_{p q} M_{n}$ are the two tangential and normal components of the vector ${ }_{p q} \mathbf{M}$ whereas ${ }_{p q} T_{t},{ }_{p q} T_{s},{ }_{p q} T_{n}$, are the two tangential and normal components of the traction vector ${ }_{p q} T_{i}=\left({ }_{p q} \sigma_{i j}+C_{i j p q}\right) n_{j}$ associated to the local problem ${ }_{p q} L$. The symmetry between the indices $p$ and q shows right away that at most six problems needs to be considered.

In similar manner the ${ }_{p} I$ problem is stated as follows: the displacements ${ }_{p} \mathbf{P}_{(\gamma)}(\mathbf{y})$ and potential ${ }_{p} Q_{(\gamma)}(\mathbf{y})$ are sought in $Y_{\gamma}$, $\gamma=1,2$, which are periodic functions of periods $w_{1}=1$, $w_{2}=\mathrm{be}^{i \Theta}, \mathrm{b}$ is the modulus of this complex number and that satisfy following equations:

${ }_{p} \sigma_{i \delta, \delta}^{(\gamma)}=0 \quad$ in $\mathrm{Y}_{\gamma}$,

${ }_{p} D_{\delta, \delta}^{(\gamma)}=0 \quad$ in $Y_{\gamma}$,

and the above mentioned interface conditions adapted to this problem

${ }_{p} \mathbf{T}^{(1)}+{ }_{p} \mathbf{T}^{(2)}=0, \quad\left[{ }_{p} Q\right]=0$, on $\Gamma, \quad{ }_{p} T_{n}^{(\gamma)}=(-1)^{\gamma+1} \tilde{K}_{n}\left[{ }_{p} P_{n}\right]$,

${ }_{p} T_{t}^{(\gamma)}=(-1)^{\gamma+1} \tilde{K}_{t}\left[P_{t}\right], \quad{ }_{p} T_{s}^{(\gamma)}=(-1)^{\gamma+1} \tilde{K}_{s}\left[p_{p} P_{s}\right]$, on $\Gamma, \quad\left[{ }_{p} D_{\delta}\right] n_{\delta}=\left[\kappa_{\delta p}^{(\gamma)}\right] \mathrm{n}_{\delta}, \quad$ on $\Gamma$,

where the functions ${ }_{p} P_{t},{ }_{p} P_{s},{ }_{p} P_{n}$ are the two tangential and normal components of the vector ${ }_{p} \mathbf{P}$ whereas ${ }_{p} T_{t},{ }_{p} T_{s},{ }_{p} T_{n}$, are the tangential and normal components of the traction vector ${ }_{p} \mathrm{~T}_{i}=\left({ }_{p} \sigma_{i j}+e_{p i j}\right) n_{j}$ associated to the local problem ${ }_{p} I$ and $\left\langle{ }_{p} \mathbf{P}\right\rangle=\left\langle{ }_{p} Q\right\rangle=0$, for the uniqueness of the solution. Furthermore,

${ }_{p} \sigma_{i \delta}^{(\gamma)}=C_{i \delta k \lambda}^{(\gamma)}{ }_{p} P_{k, \lambda}^{(\gamma)}+e_{\lambda i \delta}^{(\gamma)}{ }_{p} Q_{, \lambda}^{(\gamma)}$,

${ }_{p} D_{\delta}^{(\gamma)}=e_{\delta k \lambda}^{(\gamma)} P_{k, \lambda}^{(\gamma)}-\kappa_{\delta \lambda}^{(\gamma)}{ }_{p} Q_{, \lambda}^{(\gamma)}$.

Also the non-homogeneous ${ }_{p} I$ problems will cooperate towards the homogenized moduli.

The local equilibrium equation (14) are multiplied by test functions $v_{i}(\mathbf{y})$ and $w(\mathbf{y})$, respectively, and integrated over the 
RME domain $Y$ to obtain the weak forms of the mechanical and charge equilibrium equations:

$\int_{Y} \sigma_{i j}^{0} \frac{\partial v_{i}}{\partial y_{j}} d Y=0, \quad \int_{Y} D_{i}^{0} \frac{\partial w}{\partial y_{i}} d Y=0$.

Inserting (15) into (13) and the result into (22), and requiring that the equation hold for arbitrary $\partial u_{p}^{0} / \partial x_{q}$ and $\partial \phi^{0} / \partial x_{q}$ results in the following weak form equations for the characteristic functions, the sets of $p q$-functions ${ }_{p q} \mathbf{M}(\mathbf{y})$ and ${ }_{p q} N(\mathbf{y})$, and $p$-functions, ${ }_{p} \mathbf{P}(\mathbf{y})$ and ${ }_{p} Q(\mathbf{y})$,

$\int_{Y}\left(C_{i j k l} \frac{\partial_{p q} M_{k}}{\partial y_{l}}-e_{k i j} \frac{\partial_{p q} N}{\partial y_{k}}\right) \frac{\partial v_{i}}{\partial y_{j}} d Y=\int_{Y} C_{i j p q} \frac{\partial v_{i}}{\partial y_{j}} d Y$,

$\int_{Y}\left(e_{k i j} \frac{\partial_{p} Q}{\partial y_{k}}-C_{i j k l} \frac{\partial_{p} P_{k}}{\partial y_{l}}\right) \frac{\partial v_{i}}{\partial y_{j}} d Y=\int_{Y} e_{q i j} \frac{\partial v_{i}}{\partial y_{j}} d Y$,

$\int_{Y}\left(e_{i k l} \frac{\partial_{p q} M_{k}}{\partial y_{l}}+\kappa_{i k} \frac{\partial_{p q} N}{\partial y_{k}}\right) \frac{\partial w}{\partial y_{i}} d Y=\int_{Y} e_{i p q} \frac{\partial w}{\partial y_{i}} d Y$,

$\int_{Y}\left(\kappa_{i k} \frac{\partial_{p} Q}{\partial y_{k}}+e_{i k l} \frac{\partial_{p} P_{k}}{\partial y_{l}}\right) \frac{\partial w}{\partial y_{i}} d Y=\int_{Y} \kappa_{i q} \frac{\partial w}{\partial y_{i}} d Y$.

Eq. (23) represents a system of partial differential equations that must be solved to obtain the microscale characteristic functions ${ }_{p q} \mathbf{M}(\mathbf{y}),{ }_{p q} N(\mathbf{y}),{ }_{p} \mathbf{P}(\mathbf{y})$ and ${ }_{p} Q(\mathbf{y})$. The characteristic functions are subject to periodic boundary conditions over the RME domain $Y$ in analogous form to Pobedria [22].

The constitutive relations of the linear piezoelectric theory for a heterogeneous and periodic medium, $\Omega$, is characterized by the $Y$-periodic functions $\mathbf{C}(\mathbf{y}), \mathbf{e}(\mathbf{y}), \boldsymbol{\kappa}(\mathbf{y})$. The original constitutive relations with rapidly oscillating material coefficients are transformed in new physical relations with constant coefficients $\mathbf{C}^{*}, \mathbf{e}^{*}$, $\boldsymbol{\kappa}^{*}$ which represent the elastic, piezoelectric and permittivity properties, respectively of an equivalent homogeneous medium and are called the effective coefficients of $\Omega$. Therefore, the system (4) can be transformed into equivalent system with constant coefficients which represent the overall properties of the composite.

The main problem to obtain such average formulae is to find the $Y$-periodic solutions of nine ${ }_{p q} L,{ }_{p} I(p, q=1,2,3)$ local problems on $Y$ in terms of the fast variable $\mathbf{y}$ as it was reported by BravoCastillero et al. [10] and Sabina et al. [11] based on the mathematical statement of both problems.

Once the local problems are solved, the homogenized moduli $C_{i j p q}^{*}, e_{k i j}^{*}, \kappa_{i k}^{*}$ may be determined by using the following formulae:

$C_{i j p q}^{*}=\left\langle C_{i j p q}+C_{i j k l ~ p q} M_{k, l}+e_{k i j p q} N_{, k}\right\rangle, \quad e_{i p q}^{*}=\left\langle e_{i p q}+e_{i k l} p q M_{k, l}-\kappa_{i k} p q N_{, k}\right\rangle$, $e_{p i j}^{*}=\left\langle e_{p i j}+C_{i j k l} P_{k, l}+e_{k i j} Q_{, k}\right\rangle, \quad \kappa_{i p}^{*}=\left\langle\kappa_{i p}-e_{i k l} P_{k, l}+\kappa_{i k} Q_{, k}\right\rangle$.

Finally, the homogenized boundary value problem associated with (4)-(5) has the form

$\bar{\sigma}_{i j, j}^{0}=0, \quad \bar{D}_{i, i}^{0}=0$ in $\Omega, \quad \bar{u}_{i}^{0}=\bar{u}_{i} ; \quad \bar{\sigma}_{i j}^{0} n_{j}=\bar{S}_{i} ; \quad \bar{\phi}^{0}=\phi_{0} ; \quad \bar{D}_{i}^{0} n_{i}=0$ on $\partial \Omega$,

where the corresponding macroscale constitutive equations are

$\bar{\sigma}_{i j}^{0}=C_{i j k l}^{*} u_{k, l}^{0}+e_{k i j}^{*} \phi_{, k}^{0}, \quad \bar{D}_{i}^{0}=e_{k i j}^{*} u_{k, l}^{0}-\kappa_{i k}^{*} \phi_{, k}^{0}$.

Here $u_{i}^{0}(\mathbf{x})=\left\langle u_{i}(\mathbf{x}, \mathbf{y})\right\rangle$ is the averaged displacement vector and $\bar{\phi}^{0}(\mathbf{x})=\langle\phi(\mathbf{x}, \mathbf{y})\rangle$ the averaged electric potential.

Each local problem (16) and (17) and (19) and (20) $(p, q=1,2,3)$ uncouples into two sets of equations. The plane and antiplanestrain systems of equations which correspond to five plane-strain local problems ${ }_{p p} L,{ }_{12} L,{ }_{3} I$ and fourth antiplane-strain one ${ }_{13} L,{ }_{23} L,{ }_{1} I,{ }_{2} I$. Table 1 shows the correspondence between the effective properties and the local problems. The global behavior of the piezoelectric composite is related to the class symmetry monoclinic 2, see details in Royer and Dieulesaint [23], which
Table 1

Effective properties related to the local problems.

\begin{tabular}{lllllllll}
\hline${ }_{11} L$ & ${ }_{22} L$ & ${ }_{33} L$ & ${ }_{23} L$ & ${ }_{13} L$ & ${ }_{12} L$ & ${ }_{1} I$ & ${ }_{2} I$ & ${ }_{3} I$ \\
\hline$C_{1111}^{*}$ & $C_{1122}^{*}$ & $C_{1133}^{*}$ & 0 & 0 & $C_{1112}^{*}$ & 0 & 0 & $e_{311}^{*}$ \\
$C_{2211}^{*}$ & $C_{2222}^{*}$ & $C_{2233}^{*}$ & 0 & 0 & $C_{2212}^{*}$ & 0 & 0 & $e_{322}^{*}$ \\
$C_{3311}^{*}$ & $C_{3322}^{*}$ & $C_{3333}^{*}$ & 0 & 0 & $C_{3312}^{*}$ & 0 & 0 & $e_{333}^{*}$ \\
0 & 0 & 0 & $C_{2323}^{*}$ & $C_{2313}^{*}$ & 0 & $e_{132}^{*}$ & $e_{232}^{*}$ & 0 \\
0 & 0 & 0 & $C_{1323}^{*}$ & $C_{1313}^{*}$ & 0 & $e_{131}^{*}$ & $e_{231}^{*}$ & 0 \\
$C_{1211}^{*}$ & $C_{1222}^{*}$ & $C_{1233}^{*}$ & 0 & 0 & $C_{1212}^{*}$ & 0 & 0 & $e_{321}^{*}$ \\
0 & 0 & 0 & $e_{123}^{*}$ & $e_{113}^{*}$ & 0 & $\kappa_{11}^{*}$ & $\kappa_{12}^{*}$ & 0 \\
0 & 0 & 0 & $e_{223}^{*}$ & $e_{213}^{*}$ & 0 & $\kappa_{12}^{*}$ & $\kappa_{22}^{*}$ & 0 \\
$e_{311}^{*}$ & $e_{322}^{*}$ & $e_{333}^{*}$ & 0 & 0 & $e_{312}^{*}$ & 0 & 0 & $\kappa_{33}^{*}$ \\
\hline
\end{tabular}

contain 13 elastic, 8 piezoelectric and 4 dielectric independent coefficients.

The local-value problems set up in (16) and (17), (19) and (20) have been solved in the present work using the methods of a complex variable and the properties of doubly periodic elliptic and related functions with periods $w_{1}$ and $w_{2}$ as it is reported by Bravo-Castillero et al. [10] and Sabina et al. [11]. Taking into account that the rate of debonding would depend not only upon the debonding parameters, but also upon the elastic moduli of the components and the fiber volume fraction [1] in the solution of these problems, the following relations are used $\tilde{K}_{t}=K_{t} C_{44}^{(1)} / R$, $\tilde{K}_{n}=K_{n} C_{44}^{(1)} / R, \tilde{K}_{s}=K_{s} C_{44}^{(1)} / R$ where $K_{t}, K_{n}$ and $K_{s}$ are dimensionless parameters.

\section{Solution of antiplane problems}

Now, the problem ${ }_{13} L$ is explained in detail from the set of antiplane problems ${ }_{13} L,{ }_{23} L,{ }_{1} I,{ }_{2} I$. From now on, the preindices are not used and the effective properties are denoted with the short notation. The determination of the shear piezoelectric effective properties, denoted by $C_{44}^{*}, C_{45}^{*}, C_{55}^{*}$, (shear moduli), $e_{15}^{*}, e_{14}^{*}, e_{24}^{*}$, (shear stress piezoelectric coefficient) and $\kappa_{11}^{*}$, $\kappa_{12}^{*}, \kappa_{22}^{*}$, (transverse permittivity constant) is the main aim of this part where the constituents of each phase of the composite are of class $6 \mathrm{~mm}$ and the short indicial notation is used. In this case the relevant constitutive relations are

$\sigma_{23}=2 C_{44} \varepsilon_{23}-e_{15} E_{2}, \sigma_{13}=2 C_{44} \varepsilon_{13}-e_{15} E_{1}$,

$D_{1}=2 e_{15} \varepsilon_{23}+\kappa_{11} E_{1}, D_{2}=2 e_{15} \varepsilon_{13}+\kappa_{11} E_{2}$.

The displacement $M \equiv_{13} M$ and potential $N \equiv_{13} N$, which appear in (24), are the unique solution of the above mentioned local problem ${ }_{13} L$. In this case Eq. (16) yields

$\Delta M^{(\gamma)}=0, \quad \Delta N^{(\gamma)}=0$ in $Y_{\gamma}$

where $\Delta$ is the two-dimensional Laplacian and the contact conditions (17) on $\Gamma$ are written in the form

$T_{s}^{(1)}+T_{s}^{(2)}=0$ on $\Gamma, \quad\|N\|=0, \quad\left\|\left(e_{15} M_{, \delta}-\kappa_{11} N_{, \delta}\right) n_{\delta}\right\|=-\left\|e_{15}\right\| n_{1}$ on $\Gamma$,

$$
\left(C_{44}^{(\gamma)} M_{, \delta}^{(\gamma)}+e_{15}^{(\gamma)} N_{, \delta}^{(\gamma)}\right) n_{\delta}+C_{44}^{(\gamma)} n_{1}=(-1)^{\gamma+1} K_{s} C_{55}^{(1)}\|M\| R^{-1} \text { on } \Gamma \text {. }
$$

Eq. (24) are transformed to area integrals applying Green's theorem. The doubly periodic boundary conditions on $Y$ and the continuity of displacement and potential on $\Gamma$ leads to

$C_{55}^{*}-i C_{45}^{*}=\left\langle C_{55}\right\rangle+(-1)^{\gamma} \frac{C_{55}^{(\gamma)}}{V} \int_{\Gamma} M^{(\gamma)} d y_{2}+i M^{(\gamma)} d y_{1}+(-1)^{\gamma} \frac{e_{15}^{(\gamma)}}{V} \int_{\Gamma} N^{(\gamma)} d y_{2}+i N^{(\gamma)} d y_{1}$,

$e_{15}^{*}-i e_{14}^{*}=\left\langle e_{15}\right\rangle+(-1)^{\gamma} \frac{e_{15}^{(\gamma)}}{V} \int_{\Gamma} M^{(\gamma)} d y_{2}+i M^{(\gamma)} d y_{1}+(-1)^{\gamma} \frac{\kappa_{11}^{(\gamma)}}{V} \int_{\Gamma} N^{(\gamma)} d y_{2}+i N^{(\gamma)} d y_{1}$

where summation convention is understood for $\gamma$, which run from 1 to 2 . 
Methods of potential theory are used to solve (29). Doubly periodic harmonic functions are to be found in terms of the following Laurent and Taylor expansions of harmonic functions:

$M^{(1)}(z)=\operatorname{Re}\left\{\frac{z}{R} a_{0}+\sum_{p=1}^{\infty} 0\left(\frac{R}{z}\right)^{p} a_{p}+\sum_{k=1}^{\infty} 0 \sum_{p=1}^{\infty} 0\left(\frac{z}{R}\right)^{p} \eta_{k p} a_{k}\right\}$,

$N^{(1)}(z)=\operatorname{Re}\left\{\frac{z}{R} b_{0}+\sum_{p=1}^{\infty} 0\left(\frac{R}{z}\right)^{p} b_{p}+\sum_{k=1}^{\infty} 0 \sum_{p=1}^{\infty} 0\left(\frac{z}{R}\right)^{p} \eta_{k p} b_{k}\right\}$, in $Y_{1}$

$M^{(2)}(z)=\operatorname{Re}\left\{\sum_{p=1}^{\infty}{ }^{0} c_{p}\left(\frac{z}{R}\right)^{p}\right\}, N^{(2)}(z)=\operatorname{Re}\left\{\sum_{p=1}^{\infty}{ }^{0} d_{p}\left(\frac{z}{R}\right)^{p}\right\}$, in $Y_{2}$

where

$\eta_{k l}=-\frac{(k+l-1) !}{(k-1) ! ! !} R^{k+l} \sum_{m=-\infty}^{\infty} \sum_{n=-\infty}^{\infty} \frac{1}{\left(m w_{1}+n w_{2}\right)^{k+l}}, \quad m^{2}+n^{2} \neq 0, \quad k+l>2$

and $a_{n}, b_{n}, c_{n}, d_{n}$ are real undetermined coefficients; $w_{1}, w_{2}$, are the periods of the parallelogram array, respectively (see Fig. 2). The superscript "o" next to the summation symbol means that " $p$ " runs only over odd integers so that each term in (31) has the same antisymmetry property as $M^{(\gamma)}$ and $N^{(\gamma)}$, namely, $M^{(\gamma)}(-z)=-M^{(\gamma)}(z)$, $N^{(\gamma)}(-z)=-N^{(\gamma)}(z)$ (see more details in the works Bravo-Castillero et al. [10] and Sabina et al. [11])

The line integrals in (30) and the assumed expansions (31) produce a very simple result as a consequence of the orthogonality of the trigonometric functions, namely

$\int_{\Gamma} M^{(1)} d x_{2}+i M^{(1)} d x_{1}=\pi R\left(\bar{a}_{1}+a_{0}+\sum_{k=1}^{\infty}{ }^{o} \eta_{k 1} a_{k}\right)$,

$\int_{\Gamma} N^{(1)} d x_{2}+i N^{(1)} d x_{1}=\pi R\left(\bar{b}_{1}+b_{0}+\sum_{k=1}^{\infty}{ }^{o} \eta_{k 1} b_{k}\right)$,

$\int_{\Gamma} M^{(2)} d x_{2}+i M^{(2)} d x_{1}=\pi R c_{1}, \quad \int_{\Gamma} N^{(2)} d x_{2}+i N^{(2)} d x_{1}=\pi R d_{1}$.

Replacing (32) into Eq. (30) and taking into consideration the imperfect contact condition (29) we obtain the final expression of the effective coefficients,

$C_{55}^{*}-i C_{45}^{*}=C_{55}^{(1)}\left(1-2 V_{2} \Pi_{11}\right), e_{15}^{*}-i e_{14}^{*}=\sqrt{C_{55}^{(1)} \kappa_{11}^{(1)}}\left(E^{(1)}-2 V_{2} \Pi_{21}\right)$,

where

$\Pi_{11}=\bar{a}_{1}+E \bar{b}_{1}, \quad \Pi_{21}=E \bar{a}_{1}-\bar{b}_{1}, \quad E^{(\gamma)}=e_{15}^{(\gamma)} / \sqrt{C_{55}^{(\gamma)} \kappa_{11}^{(\gamma)}}$,

the overbar denotes complex conjugate numbers, the fiber volume fraction is $V_{2}=\pi R^{2} / V, V=\left|w_{1}\right|\left|w_{2}\right| \sin \theta$ denotes the area of periodic cell. The unknown constants $\mathrm{a}_{1}, \mathrm{~b}_{1}$ are solutions of the infinite systems related to the local problems ${ }_{13} \mathrm{~L}$, in which only the residue of $\mathrm{M}^{(\gamma)}$ and $\mathrm{N}^{(\gamma)}$ contributes towards $C_{55}^{*}, C_{45}^{*}$ and $e_{15}^{*}, e_{14}^{*}$. Thus, expressions for $a_{1}, b_{1}$ are now sought from the system of infinite equations

$\mathcal{M} \times \mathcal{D}=\mathcal{U}$,

where the vector $\mathcal{D}^{T}=\left(x_{1}, x_{2}, x_{3}, x_{4}\right)$ contains the real and imaginary parts of the unknowns $a_{1}=x_{1}+i x_{2}, \quad b_{1}=x_{3}+i x_{4}$ and the vector $\mathcal{U}$ is given by $\mathcal{U}^{T}=R\left(\beta_{21}, 0, \beta_{41}, 0\right)$. The super index $T$ denotes transpose and the $4 \times 4$-order matrix $m\left(m_{n k}\right)$ is defined by the following matrix form,

$\mathcal{M}=\mathcal{K}+R^{2} g-n_{1} \mathcal{P}^{-1} n_{2}$,
$K=\left(\begin{array}{cccc}\beta_{11} & 0 & \alpha_{11} & 0 \\ 0 & \beta_{11} & 0 & \alpha_{11} \\ \beta_{31} & 0 & \alpha_{31} & 0 \\ 0 & \beta_{31} & 0 & \alpha_{31}\end{array}\right)$

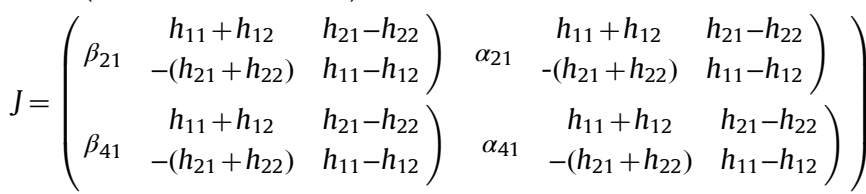

with

$h_{11}=\Re e\left\{\frac{\bar{\delta}_{1} \bar{w}_{2}-\bar{\delta}_{2} \bar{w}_{1}}{w_{1} \bar{w}_{2}-w_{2} \bar{w}_{1}}\right\}, h_{12}=\Re e\left\{\frac{\delta_{1} \bar{w}_{2}-\delta_{2} \bar{w}_{1}}{w_{1} \bar{w}_{2}-w_{2} \bar{w}_{1}}\right\}$,

$h_{21}=\Im m\left\{\frac{\bar{\delta}_{1} \bar{w}_{2}-\bar{\delta}_{2} \bar{w}_{1}}{w_{1} \bar{w}_{2}-w_{2} \bar{w}_{1}}\right\}, h_{22}=\Im m\left\{\frac{\delta_{1} \bar{w}_{2}-\delta_{2} \bar{w}_{1}}{w_{1} \bar{w}_{2}-w_{2} \bar{w}_{1}}\right\}$,

$\delta_{\gamma}=2 \zeta\left(w_{\gamma} / 2\right), \zeta(z)$

is the Zeta quasi-periodic Weierstrass function defined as

$\left.\zeta(z)=\frac{1}{z}+\sum_{m, n}^{\infty} \frac{1}{z-T_{m n}}+\frac{1}{T_{m n}}+\frac{z}{T_{m n}^{2}}\right), T_{m n}=m w_{1}+n w_{2}$

and the prime over the summation symbol means that the pair $(\mathrm{m}$, $\mathrm{n})=(0,0)$ is excluded. The Legendre's relationship links $\delta_{1}, \delta_{2}$ and the periods $w_{1}, w_{2}: \delta_{1} w_{2}-\delta_{2} w_{1}=\pi i$. The Laurent series expansion of $\zeta$ is $\zeta(z)=(1 / z)-\sum_{k=2}^{\infty} c_{k}\left(z^{2 k-1} / 2 k-1\right)$, where $c_{1}=0, c_{2}=3$ $S_{4}, c_{3}=5 S_{6}$ and $c_{k}=(3 /(2 k+1)(k-3)) \sum_{m=2}^{k-2} c_{m} c_{k-m}, \quad k \geq 4$. The lattice $S_{k}$ is defined by $S_{k}=\sum_{m, n}\left(m w_{1}+n w_{2}\right)^{-k}, m^{2}+n^{2} \neq 0, k>2$, $S_{2}=0$. In particular $S_{4}$ and $S_{6}$ used in the numerical implementation are reported in Table 1 of Chih-Bing [24] for parallelogram and rhombic cells respectively.

The matrices $n_{1}, \mathcal{P}$ and $n_{2}$ are of infinite order and for the numerical implementation it is necessary to truncate to certain order $n \in \mathbb{N}$. The matrix $\mathcal{P}=\left(\begin{array}{ccc}\mathcal{P}_{11} & \ldots & \mathcal{P}_{1 n} \\ \vdots & \ldots & \vdots \\ \mathcal{P}_{n 1} & \ldots & \mathcal{P}_{n n}\end{array}\right)_{4 n \times 4 n} \quad$ is composed of submatrices $\left(\mathcal{P}_{t s}\right)_{4 \times 4}$, defined by $\mathcal{P}_{t s}=\delta_{t s} \mathcal{K}+z_{t s}$,

$\mathcal{K}=\left(\begin{array}{cccc}\beta_{1} 2 t+1 & 0 & \alpha_{1} 2 t+1 & 0 \\ 0 & \beta_{12 t+1} & 0 & \alpha_{12 t+1} \\ \beta_{32 t+1} & 0 & \alpha_{3} 2 t+1 & 0 \\ 0 & \beta_{32 t+1} & 0 & \alpha_{32 t+1}\end{array}\right)$

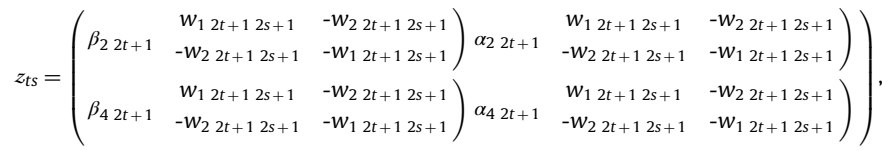

$w_{1 k p}=\Re e\left(w_{k p}\right), w_{2 k p}=\Im m\left(w_{k p}\right)$,

are the real and imaginary parts of the complex number

$w_{k p}=\frac{(k+p-1) !}{(k-1) !(p-1) !} \frac{R^{k+p}}{\sqrt{k p}} S_{k+p}, k=2 t-1, p=2 s-1, t, s=1,2,3 \ldots$

The matrices $n_{1}=\left(\begin{array}{lll}n_{41} & \cdots & n_{4 n}\end{array}\right)_{4 \times 4 n}$ and $n_{2}=\left(\begin{array}{c}n_{14} \\ \vdots \\ n_{n 4}\end{array}\right)_{4 n \times 4}$ are composed of sub-matrices $\left(n_{4 t}\right)_{4 \times 4}$ and $\left(n_{t 4}\right)_{4 \times 4}$ defined by $n_{4 k}=z_{2 t+11}, \quad n_{t 4}=z_{12 t+1} \quad$ respectively. The magnitudes $\beta_{1 p}, \beta_{2 p}, \beta_{3 p}, \beta_{4 p}, \alpha_{1 p}, \alpha_{2 p}, \alpha_{3 p}, \alpha_{4 p}$ are given as follows,

$\beta_{1 p}=1, \quad \beta_{2 p}=\frac{1-\chi_{p}\left(1-K_{s}^{-1} p\right)}{1+\chi_{p}\left(1+K_{s}^{-1} p\right)}, \quad \beta_{3 p}=1, \quad \beta_{4 p}=\frac{E^{(1)}-\sqrt{\chi_{p} \chi_{t}} E^{(2)}\left(1-K_{s}^{-1} p\right)}{E^{(1)}+\sqrt{\chi_{p} \chi_{t}} E^{(2)}\left(1+K_{s}^{-1} p\right)}$,

$\alpha_{1 p}=\frac{E^{(1)}+\chi_{p} E^{(1)} p K_{s}^{-1}+\sqrt{\chi_{p} \chi_{s}} E^{(2)}}{1+\chi_{p}\left(1+K_{s}^{-1} p\right)}, \quad \quad \alpha_{2 p}=\frac{E^{(1)}+\chi_{p} E^{(1)} p K_{s}^{-1}-\sqrt{\chi_{p} \chi_{t}} E^{(2)}}{1+\chi_{p}\left(1+K_{s}^{-1} p\right)}$,

$\alpha_{3 p}=\frac{-1+p \sqrt{\chi_{p} \chi_{\chi}} E^{(1)} E^{(2)} K_{s}^{-1}-\chi_{t}}{E^{(1)}+\sqrt{\chi_{p} \chi_{t}} E^{(2)}\left(1+K_{s}^{-1} p\right)}, \quad \quad \alpha_{4 p}=\frac{-1+p \sqrt{\chi_{p} \chi_{t}} E^{(1)} E^{(2)} K_{s}^{-1}+\chi_{t}}{E^{(1)}+\sqrt{\chi_{p} \chi_{t}} E^{(2)}\left(1+K_{s}^{-1} p\right)}$, 
where

$\chi_{p}=C_{44}^{(2)} / C_{44}^{(1)}, \chi_{t}=\kappa_{11}^{(2)} / \kappa_{11}^{(1)}$.

The limit case of perfect contact condition for piezoelectric antiplane problem is derived as a particular case of (34)-(36) as $\mathrm{K}_{\mathrm{s}} \rightarrow \infty$. In this case, the parameters $\mathrm{a}_{1}, \mathrm{~b}_{1}$ are the same that formula (3.25) page 1475 reported by Bravo-Castillero et al. [10]. The infinite system (34)-(36) is used such that it is truncated for obtaining an $n \times n$ order system. It is interesting to note that the effective properties are monotonic function of order $n$ of the solution of the system. The numerical results converge well to the exact solutions when an adequate order in the solution of the system is chosen as $n$ increase. The truncation order for solving the system increases as the parameters $\mathrm{K}, \chi^{*}$ and the fiber volume fraction are high. In the numerical examples the solutions are given for $n=10$, because this order of $n$ achieves the require accuracy for the parameters used.

The remaining antiplane problems ${ }_{23} L,{ }_{\alpha} I(\alpha=1,2)$ can be solved in analogous form to the aforementioned problem. As a summary, all the effective coefficients derived from the antiplane set of local problems can be listed as follows:

$C_{55}^{*}-i C_{45}^{*}=C_{55}^{(1)}\left(1-2 V_{2} H_{11}\right)$,

$C_{45}^{*}-i C_{44}^{*}=-C_{55}^{(1)}\left(i+2 V_{2} H_{12}\right)$,

$e_{15}^{*}-i e_{14}^{*}=\sqrt{C_{55}^{(1)} \kappa_{11}^{(1)}}\left(E-2 V_{2} H_{21}\right)$,

$e_{14}^{*}-i e_{24}^{*}=-\sqrt{C_{55}^{(1)} \kappa_{11}^{(1)}}\left(i E+2 V_{2} H_{22}\right)$,

$\kappa_{11}^{*}-i \kappa_{12}^{*}=\kappa_{11}^{(1)}\left(1+2 V_{2} H_{31}\right)$,

$\kappa_{12}^{*}-i \kappa_{22}^{*}=-\kappa_{11}^{(1)}\left(i-2 V_{2} H_{31}\right)$

where

$\begin{array}{ll}H_{1 \alpha}=\bar{a}_{1(\alpha 3)}+E \bar{b}_{1(\alpha 3)}, & H_{2 \alpha}=E \bar{a}_{1(\alpha 3)}-\bar{b}_{1(\alpha 3)}, \\ H_{3 \alpha}=E \bar{a}_{1(\alpha)}-\bar{b}_{1(\alpha)}, & E=e_{15}^{(1)} / \sqrt{C_{55}^{(1)} \kappa_{11}^{(1)}},\end{array}$

the over bar denotes complex conjugate numbers and $a_{1(\alpha 3)}, b_{1(\alpha 3)}$, $a_{1(\alpha)}$ and $b_{1(\alpha)}$ are solution of the infinite systems related to the local problems ${ }_{13} L,{ }_{23} L,{ }_{1} I$ and ${ }_{2} I$

\section{Solution of plane local problems}

Now, the problem ${ }_{\beta \beta} L$ is considered. We can obtain from (18) the constitutive equations for the plane piezoelectric problem

$$
\sigma_{11}=C_{11 \beta \beta} M_{1,1}+C_{12 \beta \beta} M_{2,2}, \quad \sigma_{22}=C_{12 \beta \beta} M_{1,1}+C_{11}{ }_{\beta \beta} M_{2,2} \text {, }
$$$$
\sigma_{12}=C_{66}\left({ }_{\beta \beta} M_{1,2}+{ }_{\beta \beta} M_{2,1}\right), \quad D_{3}=e_{15}{ }_{\beta \beta} M_{1,1}+e_{24}{ }_{\beta \beta} M_{2,2} \text {. }
$$

The system of equations related to this plane problem is decoupled into two pure elastic equations $\sigma_{11,1}+\sigma_{12,2}=$ $0, \sigma_{12,1}+\sigma_{22,2}=0$, and one electric equation $D_{3,1}+D_{3,2}=0$ with the same unknown functions ${ }_{\beta \beta} M_{1},{ }_{\beta \beta} M_{2}$. The $\beta \beta$ pre-subindices are dropped from all relevant quantities. Therefore, we only need to find the solution of the same problem derived from plane elasticity equations with imperfect contact condition

$\sigma_{\alpha \delta, \delta}^{(\gamma)}=0$ in $Y_{\gamma}, \quad T_{n}^{(1)}+T_{n}^{(2)}=0, \quad T_{t}^{(1)}+T_{t}^{(2)}=0$ on $\Gamma$,

$T_{n}^{(\gamma)}=(-1)^{\gamma+1} C_{66}^{(1)} K_{n}\left\|M_{n}\right\| R^{-1}, \quad T_{t}^{(\gamma)}=(-1)^{\gamma+1} C_{66}^{(1)} K_{t}\left\|M_{t}\right\| R^{-1}$ on $\Gamma$.

Now, the idea consists to rewrite the mathematical formulation of imperfect contact given by (39) in terms of the potential functions $\varphi_{\gamma}, \psi_{\gamma}$.

The methods of a complex variable $z$ in terms of two harmonic functions and the Kolosov-Muskhelishvili complex potentials are applicable. The potentials are related to the displacement and stress components by means of the formulae

$2 C_{66}^{(\gamma)}\left(u_{1}^{(\gamma)}+i u_{2}^{(\gamma)}\right)=\chi^{(\gamma)} \varphi_{\gamma}(z)-\overline{z \varphi_{\gamma}^{\prime}}(z)-\overline{\psi_{\gamma}}(z)$,

$\sigma_{11}^{(\gamma)}+\sigma_{22}^{(\gamma)}=2\left[\varphi_{\gamma}^{/}(z)+\overline{\varphi_{\gamma}^{\prime}}(z)\right]$,

$\sigma_{22}^{(\gamma)}-\sigma_{11}^{(\gamma)}+2 i \sigma_{12}^{(\gamma)}=2\left[z \varphi_{\gamma}^{/ /}(z)+\psi_{\gamma}^{\prime}(z)\right]$,

and $\chi^{(\gamma)}=3-4 \nu^{(\gamma)}, \nu^{(\gamma)}=C_{12}^{(\gamma)} /\left(C_{11}^{(\gamma)}+C_{12}^{(\gamma)}\right)$ is the transverse Poisson's ratio. The prime denotes a derivative with respect to $z$. The representation of the complex potentials $\varphi_{\gamma}, \psi_{\gamma}$ of periods $\omega_{\gamma}$ is given in the form

$\varphi_{1}(z)=\frac{a_{0}}{R} z+\varsigma(z) R a_{1}+\sum_{k=3}^{\infty} * R_{k} a_{k} \sum_{m, n}^{\infty}\left(z-\beta_{m n}\right)^{-k}$,

$\psi_{1}(z)=\frac{z}{R} b_{0}+\varsigma(z) R b_{1}+Q(z) R a_{1}+\sum_{k=3}^{\infty} *\left[R^{k} b_{k} \sum_{m, n}^{\infty}\left(z-\beta_{m n}\right)^{-k}+k R^{k} a_{k} \sum_{m, n}^{\infty} \bar{\beta}_{m n}\left(z-\beta_{m n}\right)^{-k-1}\right]$

$\varphi_{2}(z)=\sum_{k=1}^{\infty} *\left(\frac{z}{R}\right)_{k} c_{k}, \quad \psi_{2}(z)=\sum_{k=1}^{\infty} *\left(\frac{z}{R}\right)_{k} d_{k}$,

where the coefficients $a_{0}, b_{0}, a_{k}, b_{k} c_{k} d_{k}$ are complex numbers and undetermined, $Q(z)$ is Natanzon's function, $\beta_{m n}=m w_{1}+n w_{2}$, $w_{1}=1, w_{2}=\operatorname{Re}^{i \theta}$ for $m, n \in \mathbb{Z}$, the asterisk on the sigma symbol means that the double summation excludes the term $m=n=0$. The double periodicity and quasi-periodicity of these functions leads to

$\chi^{(1)} a_{0}-\bar{a}_{0}=\left(-A_{1} \chi^{(1)} a_{1}+\bar{A}_{1} \bar{a}_{1}+\bar{A}_{2} \bar{b}_{1}\right) R^{2}, \quad b_{0}=\left[\bar{A}_{2} \chi^{(1)} \bar{a}_{1}+A_{3} a_{1}-A_{1} b_{1}\right] R^{2}$,

where

$A_{1}=\frac{\bar{w}_{1} \delta_{2}-\bar{w}_{2} \delta_{1}}{\bar{w}_{1} w_{2}-w_{1} \bar{w}_{2}}, \quad A_{2}=\frac{w_{1} \delta_{2}-w_{2} \delta_{1}}{\bar{w}_{1} w_{2}-w_{1} \bar{w}_{2}}, \quad A_{3}=\frac{\bar{w}_{1} P_{2}-\bar{w}_{2} P_{1}}{\bar{w}_{1} w_{2}-w_{1} \bar{w}_{2}}$,

$P_{\alpha}=2 Q\left(\frac{W_{\alpha}}{2}\right)-\bar{w}_{\alpha} \wp\left(\frac{W_{\alpha}}{2}\right), \quad \wp(z)=-\zeta^{\prime}(z)$

Using the simple action-reaction principle given by the second and third equation (39) and after some algebraic manipulations of the formulae Kolosov-Muskhelishvili (40) and the series expansion of the potential functions (41) we can obtain the following relations between the unknown constants of the above expansions:

$b_{1}=\frac{2 C}{B} \Re e\left\{-R^{2} A_{1} a_{1}+\sum_{k=1}^{\infty}{ }^{o} \eta_{k 1} a_{k}\right\}-\frac{P}{B} R \gamma_{2 \beta}$,

$b_{p+2}=\left[p-\frac{D_{p}}{E_{p}} K_{n} K_{t} \chi_{m}\left(\kappa_{1}+1\right)\right] a_{p}-\left[1+\frac{B_{p}}{E_{p}} K_{n} K_{t} \chi_{m}\left(\kappa_{1}+1\right)\right] \sum_{k=1}^{\infty}{ }^{o} \bar{\eta}_{k p+2} \bar{a}_{k}$,

$c_{1}=\frac{1}{2\left(\kappa_{2}+1\right)}\left(\begin{array}{c}-C_{1}^{+} A_{1} R^{2} a_{1}-C_{1}^{-} \bar{A}_{1} R^{2} \bar{a}_{1} \\ +C_{1}^{+} \sum_{k=1}^{\infty}{ }^{o} \eta_{k 1} a_{1} \\ +C_{1}^{-} \sum_{k=1}^{\infty}{ }^{o} \bar{\eta}_{k 1} \bar{a}_{1}+\left(\chi^{(2)}+1-2 \beta_{0} \frac{P}{B}\right) R \gamma_{2 j}\end{array}\right)$

$c_{p+2}=-\frac{K_{n} K_{t} \chi_{m}\left(\kappa_{1}+1\right)}{E_{p}}\left(D_{p} \bar{a}_{p}+B_{p} \sum_{k=1}^{\infty}{ }^{o} \eta_{k p+2} a_{k}\right)$,

$$
\begin{aligned}
d_{p}= & \left.K_{n} K_{t} \chi_{m}\left(\kappa_{1}+1\right) \frac{C_{p}}{E_{p}} \bar{a}_{p}+\frac{A_{p}}{E_{p}} \sum_{k=1}^{\infty}{ }^{0} \eta_{k p+2} a_{k}\right) \\
& +\frac{C_{p}\left(K_{n}-K_{t}\right) \gamma_{3 j}-A_{p}\left(K_{n}+K_{t}\right) \gamma_{3 j}+K_{n} K_{t} C_{p} \gamma_{1 j}}{E_{p}} \chi_{m} R \delta_{1 p},
\end{aligned}
$$

where

$B=\left(1-\chi_{m}-\frac{2 p \chi_{m}}{K_{n}}\right)\left(A_{p}^{0}\right)^{-1}, \quad C=B \frac{1-\kappa_{2}+\chi_{m}\left(\kappa_{1}-1\right)}{2 \alpha_{0}}+\frac{2 \chi_{m}}{\alpha_{0} K_{n}}$,

$P=B\left(\frac{\kappa_{2}-1}{2 \alpha_{0}}-\frac{4 \chi_{m}\left(1+\gamma_{4 \beta} / \gamma_{2 \beta}\right)}{2 \alpha_{0} K_{n}}\right), \quad C_{1}^{ \pm}=1+\kappa_{2} \pm \chi_{m}\left(1+\kappa_{1}\right)+2 \beta_{0} \frac{C}{B}$, 
$\alpha_{0}=\chi_{m}\left[1-\mathfrak{R} e\left\{A_{2}\right\} R^{2}\right]+\left(\kappa_{2}-1-\frac{4 \chi_{m}}{K_{n}}\right)\left[\frac{\mathfrak{R} e\left\{\mathrm{~A}_{2}\right\} R^{2}}{\kappa_{1}-1}+\frac{1}{2}\right]$,

$\beta_{0}=\frac{\left(\kappa_{2}+1\right) \operatorname{Re}\left\{\mathrm{A}_{2}\right\} R^{2}}{\kappa_{1}-1}-i \chi_{m} \Im m\left\{A_{2}\right\} R^{2}+\frac{\left(\kappa_{2}+1\right)}{2}$,

$\mathfrak{R} e\{z\}$ and $\mathfrak{I} m\{z\}$ denote real and imaginary part of complex number $z$. Then,

$A_{p}^{0}=\chi_{m} \kappa_{1}+1-\frac{2 p \chi_{m}}{K_{n}}+K_{n} K_{t} \chi_{m}\left(\kappa_{1}+1\right)\left(\kappa_{2}+\chi_{m}-\frac{2(p+2) \chi_{m}}{K_{n}}\right) \frac{D_{p}}{E_{p}}$,

$A_{p}=(p+2)\left(K_{n} K_{t}\left(1-\chi_{m}\right)+p\left(K_{n}-K_{t}\right) \chi_{m}+\left(K_{n}+K_{t}\right) \chi_{m}\right)$,

$B_{p}=K_{t} K_{n}\left(1-\chi_{m}\right)+p\left(K_{n}-K_{t}\right) \chi_{m}$,

$C_{p}=K_{n} K_{t}\left(\kappa_{2}+\chi_{m}\right)+(p+2) \chi_{m}\left(\left(K_{n}+K_{t}\right) p+K_{n}-K_{t}\right)$,

$D_{p}=p \chi_{m}\left(K_{n}+K_{t}\right), E_{p}=A_{p} D_{p}-C_{p} B_{p}$,

$\delta_{i k}$ is the Kronecker's delta function, $C_{k}^{n}$ is the binomial coefficient, $V_{1}$ and $V_{2}$ are the area of the matrix and fiber respectively, $V_{1}+V_{2}=1$. The parameters involved in this expression are defined as follows:

$\eta_{k p}=-\sqrt{\frac{k}{p}} w_{k p}, \quad T_{n+k}=\sum_{p, q} \frac{p \bar{w}_{1}+q \bar{w}_{2}}{\left(p w_{1}+q w_{2}\right)^{n+k+1}}, \quad p^{2}+q^{2} \neq 0, \quad p, q$-integer numbers.

The above expressions (43)-(47) depend on the unknown parameter $a_{p}$ which can be calculated from the following system of algebraic equations:

$a_{p}+H_{1 p} a_{1}+H_{2 p} \bar{a}_{1}+\sum_{k=1}^{\infty}{ }^{0} W_{k p} a_{k}+\sum_{k=1}^{\infty}{ }^{0} M_{k p} \bar{a}_{k}=H_{3 p} R \gamma_{2 j}$,

where $p=1,3,5, \ldots$,

$H_{1 p}=B A_{2} \chi^{(1)} R^{2} \delta_{1 p}-\left(\bar{\eta}_{1 p}-\bar{A}_{1} R^{2} \delta_{1 p}\right) R^{2} A_{1} C, H_{2 p}=B \bar{A}_{3} R^{2} \delta_{1 p}-\left(\bar{\eta}_{1 p}-\bar{A}_{1} R^{2} \delta_{1 p}\right) R^{2} \bar{A}_{1} C$,

$M_{k p}=B G_{k p}-B D_{p}^{0} \bar{\eta}_{k+2 p}+\left(\bar{\eta}_{1 p}-\bar{A}_{1} R^{2} \delta_{1 p}\right) C \bar{\eta}_{k 1}+B_{p}^{0}\left(A_{p}^{0}\right)^{-1} \bar{\eta}_{k p+2}$,

$W_{k p}=-B C_{k}^{0} r_{k p}+\left(\bar{\eta}_{1 p}-\bar{A}_{1} R^{2} \delta_{1 p}\right) C \eta_{k 1}$,

$H_{3 p}=\left(\bar{\eta}_{1 p}-\bar{A}_{1} R^{2} \delta_{1 p}\right) P+\frac{2 \chi_{m}}{K_{n}}\left(A_{p}^{0}\right)^{-1} \frac{\gamma_{3 j}}{\gamma_{2 j}} \delta_{1 p}-\left(1-\frac{2 \chi_{m} p}{K_{n}}\right)\left(A_{p}^{0}\right)^{-1} \frac{\gamma_{1 j}}{\gamma_{2 j}} \delta_{1 p}$,

$B_{p}^{0}=\chi_{m}\left(\kappa_{1}+1\right)\left[1+K_{n} K_{t}\left(\kappa_{2}+\chi_{m}-\frac{2 \chi_{m}}{K_{n}}(p+2)\right) \frac{B_{p}}{E_{p}}\right]$,

$C_{p}^{0}=1+K_{n} K_{t} \chi_{m}\left(1+\kappa_{1}\right) \frac{B_{p}}{E_{p}}, D_{p}^{0}=K_{n} K_{t} \chi_{m}\left(1+\kappa_{1}\right) \frac{D_{p}}{E_{p}}$

$r_{k p}=\sum_{i=3}^{\infty}{ }^{o} \eta_{k i} \eta_{i p}, G_{k p}=(p+2) \eta_{k(p+2)}+k \eta_{k+2 p}+k R^{p+k} C_{p+k}^{p} T_{p+k}$.

Eq. (48) represents an infinite linear system from which we can calculate coefficients $a_{p}$ as it is done for example by RodriguezRamos et al. [25] being $2 \gamma_{1}(\beta)=C_{2 \beta}^{(1)}-C_{2 \beta}^{(2)}+C_{1 \beta}^{(2)}-C_{1 \beta}^{(1)}, \quad T_{k+l}=$ $\sum_{m, n} \bar{\beta}_{m n} \beta_{m n}^{-k-l-1}$ for $k+l \geq 3$ and $\delta_{k l}$ denotes delta Kronecker's symbol.

From the solution of the in-plane local problems ${ }_{\beta \beta} L,{ }_{12} L$ and ${ }_{3} I$ the coefficients $C_{11}^{*}, C_{12}^{*}, C_{13}^{*}, C_{22}^{*}, C_{23}^{*}, C_{33}^{*}, C_{66}^{*}, C_{16}^{*}, C_{26}^{*}$, $C_{36}^{*}, e_{31}^{*}, e_{32}^{*}, e_{33}^{*}, e_{36}^{*}, \kappa_{33}^{*}$ are derived. The closed form formulae of the effective coefficients can be listed as follows:

Elastic

$$
\begin{aligned}
C_{11}^{*}= & \left\langle C_{11}\right\rangle-V_{2} \frac{\|k\|^{2}}{m_{1}} \mathfrak{R e}\left\{\kappa_{2} \Delta_{1}-\bar{\Delta}_{1}\right\}-V_{2}\|k\|\left(\kappa_{1}+1\right) \Re \mathrm{e}\left\{\frac{a_{11}}{R\|k\|}\right\}+V_{2}\|m\| \\
& +2 V_{2} \frac{k_{1}\|k\|}{m_{1} K_{n}}\left(2 \chi_{m} \mathfrak{R e}\left\{\Delta_{1}\right\}+\frac{k_{2}}{\|k\|}\right),
\end{aligned}
$$

$$
\begin{aligned}
C_{12}^{*}= & \left\langle C_{12}\right\rangle-V_{2} \frac{\|k\|^{2}}{m_{1}} \mathfrak{R e}\left\{\kappa_{2} \Delta_{2}-\bar{\Delta}_{2}\right\}+V_{2}\|k\|\left(\kappa_{1}+1\right) \Re \mathrm{e}\left\{\frac{a_{12}}{R\|k\|}\right\}-V_{2}\|m\| \\
& +2 V_{2} \frac{k_{1}\|k\|}{m_{1} K_{n}}\left(2 \chi_{m} \mathfrak{R e}\left\{\Delta_{2}\right\}+\frac{k_{2}}{\|k\|}\right),
\end{aligned}
$$$$
C_{13}^{*}=\left\langle C_{13}\right\rangle-V_{2} \frac{\|k\|\left\|C_{13}\right\|}{m_{1}} \mathfrak{R e}\left\{\kappa_{2} \Delta_{3}-\bar{\Delta}_{3}\right\}-V_{2}\left\|C_{13}\right\|\left(\kappa_{1}+1\right) \Re \mathrm{Re}\left\{\frac{a_{13}}{R\left\|C_{13}\right\|}\right\}
$$

$$
\begin{aligned}
& \left.+2 V_{2} \frac{k_{1}\left\|C_{13}\right\|}{m_{1} K_{n}} \quad 2 \chi_{m} \Re \mathrm{e}\left\{\Delta_{3}\right\}+\frac{C_{13}^{(2)}}{\left\|C_{13}\right\|}\right) \\
& C_{22}^{*}=\left\langle C_{11}\right\rangle-V_{2} \frac{\|k\|^{2}}{m_{1}} \Re \mathrm{e}\left\{\kappa_{2} \Delta_{2}-\bar{\Delta}_{12}\right\}+V_{2}\|k\|\left(\kappa_{1}+1\right) \Re \mathrm{e}\left\{\frac{a_{12}}{R\|k\|}\right\}+V_{2}\|m\| \\
& +2 V_{2} \frac{k_{1}\|k\|}{m_{1} K_{n}}\left(2 \chi_{m} \mathfrak{R e}\left\{\Delta_{2}\right\}+\frac{k_{2}}{\|k\|}\right) \\
& C_{23}^{*}=\left\langle C_{13}\right\rangle-V_{2} \frac{\|k\|\left\|C_{13}\right\|}{m_{1}} \Re \mathrm{e}\left\{\kappa_{2} \Delta_{3}-\bar{\Delta}_{3}\right\}+V_{2}\left\|C_{13}\right\|\left(\kappa_{1}+1\right) \Re \mathrm{R}\left\{\frac{a_{13}}{R\left\|C_{13}\right\|}\right\} \\
& \left.+2 V_{2} \frac{k_{1}\left\|C_{13}\right\|}{m_{1} K_{n}} \quad 2 \chi_{m} \Re \operatorname{Re}\left\{\Delta_{3}\right\}+\frac{C_{13}^{(2)}}{\left\|C_{13}\right\|}\right), \\
& C_{33}^{*}=\left\langle C_{33}\right\rangle-V_{2} \frac{\left\|C_{13}\right\|^{2}}{m_{1}} \mathfrak{R e}\left\{\kappa_{2} \Delta_{3}-\bar{\Delta}_{3}\right\} \\
& +2 V_{2} \frac{C_{13}^{(1)}\left\|C_{13}\right\|}{m_{1} K_{n}}\left(2 \chi_{m} \Re e\left\{\Delta_{3}\right\}+\frac{C_{13}^{(2)}}{\left\|C_{13}\right\|}\right), \\
& C_{66}^{*}=C_{66}^{(1)}-V_{2}\|m\|\left(\kappa_{1}+1\right) \Im \operatorname{m}\left\{a_{1(12)}\right\}, \\
& C_{61}^{*}=V_{2}\|k\| \Im \mathrm{m}\left[\left(\kappa_{1}+1\right) \frac{a_{11}}{R\|k\|}\right], \\
& C_{62}^{*}=V_{2}\|k\| \Im \mathrm{m}\left[\left(\kappa_{1}+1\right) \frac{a_{12}}{R\|k\|}\right] \text {, } \\
& C_{63}^{*}=V_{2}\left\|C_{13}\right\| \Im \mathrm{m}\left[\left(\kappa_{1}+1\right) \frac{a_{13}}{R\left\|C_{13}\right\|}\right] \text {. }
\end{aligned}
$$

Piezoelectric

$$
\begin{aligned}
e_{31}^{*}= & \left\langle e_{31}\right\rangle-V_{2} \frac{\|k\|\left\|e_{31}\right\|}{m_{1}} \mathfrak{R e}\left\{\kappa_{2} \Delta_{1}-\bar{\Delta}_{1}\right\}+2 V_{2} \frac{e_{31}^{(1)}\|k\|}{m_{1} K_{n}}\left(2 \chi_{m} \mathfrak{R e}\left\{\Delta_{1}\right\}+\frac{k_{2}}{\|k\|}\right), \\
e_{32}^{*}= & \left\langle e_{31}\right\rangle-V_{2} \frac{\|k\|\left\|e_{31}\right\|}{m_{1}} \mathfrak{R e}\left\{\kappa_{2} \Delta_{2}-\bar{\Delta}_{2}\right\}+2 V_{2} \frac{e_{31}^{(1)}\|k\|}{m_{1} K_{n}}\left(2 \chi_{m} \mathfrak{R e}\left\{\Delta_{2}\right\}+\frac{k_{2}}{\|k\|}\right), \\
e_{33}^{*}= & \left\langle e_{33}\right\rangle-V_{2} \frac{\left\|C_{13}\right\|\left\|e_{31}\right\|}{m_{1}} \mathfrak{R e}\left\{\kappa_{2} \Delta_{3}-\bar{\Delta}_{3}\right\} \\
& +2 V_{2} \frac{e_{31}^{(1)}\left\|C_{13}\right\|}{m_{1} K_{n}}\left(2 \chi_{m} \mathfrak{R e}\left\{\Delta_{3}\right\}+\frac{e_{31}^{(2)}}{\left\|C_{13}\right\|}\right),
\end{aligned}
$$

$e_{36}^{*}=-V_{2} \frac{\|m\|\left\|e_{31}\right\|}{m_{1}} \mathfrak{R e}\left\{\kappa_{2} \Delta_{12}-\bar{\Delta}_{12}\right\}+4 \chi_{m} V_{2} \frac{\|m\| e_{31}^{(1)}}{m_{1} K_{n}} \mathfrak{R e}\left\{\Delta_{12}\right\}$,

where $\Delta_{\beta}=c_{1} /\left(R \chi_{m} \gamma_{2 \beta}\right), a_{1 \beta}$ denotes the residue $a_{1}$ of the function $\varphi_{1}(z)$ in (41) for each problem ${ }_{\beta \beta} L$. Analogously, the magnitude $a_{1 \text { (12) }}$ denotes the residue $a_{1}$ of the function $\varphi_{1}(z)$ for the local plane problem $L_{12}$, where the system (48) and the expressions (49) are the same with the only different expression $H_{3}\left(h_{n}\right)=i E \delta_{1 n}$. Moreover, $\Delta_{12}=c_{1} /\left(2 R \chi_{m}\right)$.

The local problem ${ }_{3} I$ is solved analogously to the problem $L_{\beta \beta}$ and we omit the steps for the solution, only we present the analytic expression of the dielectric permittivity effective coefficient $\kappa_{33}^{*}$ in order to characterize completely the composite

Dielectric permittivity

$\left.\kappa_{33}^{*}=\left\langle\kappa_{33}\right\rangle+V_{2} \frac{\left\|e_{31}\right\|^{2}}{m_{1}} \mathfrak{R e}\left\{\kappa_{2} \Delta-\bar{\Delta}\right\}+2 V_{2} \frac{e_{31}^{(1)}\left\|e_{31}\right\|}{m_{1} K_{n}} \quad 2 \chi_{m} \mathfrak{R e}\{\Delta\}+\frac{e_{31}^{(2)}}{\left\|e_{31}\right\|}\right)$,

and $\Delta=c_{1} /\left(R \chi_{m}\left\|e_{31}\right\|\right)$.

\section{Analysis of the numerical results. Validation of the model}

To illustrate how the composite material parameters vary with imperfect interfacial parameters and volume fraction of 
piezoelectric ceramic, the material parameters of PZT-7A, $\mathrm{BaTiO}_{3}$ and Araldite D are used. They are listed in Table 2.

Moreover, as it was stated aforementioned in the calculations by AHM, the following relations are used: $\tilde{K}_{t}=K_{t} C_{44}^{(1)} / R$, $\tilde{K}_{n}=K_{n} C_{44}^{(1)} / R, \quad \tilde{K}_{s}=K_{s} C_{44}^{(1)} / R$ where $K_{t}, K_{n}$ and $K_{s}$ are dimensionless imperfect parameters.

As a validation of the present model derived using AHM, in Table 3 a comparison between the model by AHM with the model by FEM reported by Kar-Gupta and Venkatesh [26] for 1-3 piezocomposite system under perfect contact with square-cell distribution and individual constituent phases $\mathrm{BaTiO}_{3}$ matrix and PZT-7A fiber of hexagonal $6 \mathrm{~mm}$ symmetry is presented. The calculations were made for fiber volume fraction $V_{2}=0.7$. Notice a good concordance between both models.

Some numerical calculations are shown in Table 4 in order to illustrate the influence of imperfect adhesion in the behavior of the 1-3 piezocomposite with square and hexagonal cells distribution and individual constituent phases $\mathrm{BaTiO}_{3}$ matrix and PZT-7A fiber. Two different mechanical imperfect parameters are considered $K=5$ (imperfect contact) and $K=10^{12}$ (perfect contact) for two different configuration of the cells and $V_{2}=0.6$. The case of imperfect contact, where the rigidity of the interface is softening, the effective moduli decrease because the imperfection makes weaker the interaction between fiber and matrix. This situation is simulating an augment of the porosity in the composite. On the other hand, the configuration of the unit cell does not influence upon the numerical values of the effective properties because the global behavior of the composites with hexagonal or square cell is transversely isotropic material as it was shown in Bravo-Castillero et al. [10] and Sabina et al. [11]. This is different from other configuration of the cells where the symmetry of the global behavior of composite is tetragonal as it was studied in Rodriguez-Ramos et al. [14]. Other possible cause of this fact is the low contrast of the properties between matrix and fibers.

As another validation a comparison between the present model for perfect contact with EEVM reported in Guinovart-Díaz et al. [13] and Yan et al. [28] for a two-phase PZT/Epoxy composite for four different configuration of basic cell arrangements $\left(\theta=45^{\circ}, 60^{\circ}, 75^{\circ}, 90^{\circ}\right)$ is shown in Table 5. The used constituents properties are listed in Table 1 of Yan et al. [28] and the calculations were made for fiber volume fraction $V_{2}=0.6$. In this example, where the contrast between matrix and fibers is high,

Table 2

Electroelastic material constants.

\begin{tabular}{lclll}
\hline & $\begin{array}{l}\mathrm{BaTiO}_{3} \text { matrix } \\
\text { Kar-Gupta [26] }\end{array}$ & $\begin{array}{l}\text { PZT-7A fiber } \\
\text { Kar-Gupta [26] }\end{array}$ & $\begin{array}{l}\text { PZT-7A fiber } \\
\text { Levin [27] }\end{array}$ & $\begin{array}{l}\text { Araldite D matrix } \\
\text { Levin [27] }\end{array}$ \\
\hline$C_{11}(\mathrm{GPa})$ & 150.4 & 157.7 & 148 & 8.0 \\
$C_{12}(\mathrm{GPa})$ & 65.63 & 87.67 & 76.2 & 4.4 \\
$C_{13}(\mathrm{GPa})$ & 65.94 & 81.2 & 74.2 & 4.4 \\
$C_{33}(\mathrm{GPa})$ & 145.5 & 125.7 & 131 & 8.0 \\
$C_{44}(\mathrm{GPa})$ & 43.86 & 29.41 & 25.4 & 1.8 \\
$C_{66}(\mathrm{GPa})$ & 42.37 & 34.97 & 35.9 & 1.8 \\
$\kappa_{11}(\mathrm{nC} / \mathrm{Vm})$ & 12.8 & 8.23 & 4.6 & 4.2 \\
$\kappa_{33}(\mathrm{nC} / \mathrm{Vm})$ & 15.1 & 3.76 & 2.35 & 4.2 \\
$e_{31}\left(\mathrm{C} / \mathrm{m}^{2}\right)$ & -4.32 & -2.30 & -2.1 & 0 \\
$e_{33}\left(\mathrm{C} / \mathrm{m}^{2}\right)$ & 17.4 & 9.49 & 12.3 & 0 \\
$e_{15}\left(\mathrm{C} / \mathrm{m}^{2}\right)$ & 11.4 & 10.6 & 9.2 & 0 \\
\hline
\end{tabular}

the configuration of the periodic cell does have a major influence on the effective piezoelectric moduli in comparison with Table 4. Notice a good agreement of the present results derived by AHM with spring parameter $K=10^{12}$ and the results obtained by EEVM.

In Table 6 the same configuration of basic cells $\left(\theta=45^{\circ}, 60^{\circ}, 75^{\circ}, 90^{\circ}\right)$ for the PZT/Epoxy composite are considered. In this case, the influence of the imperfect spring parameter $K_{n}=K_{t}=K_{s}=K$ is illustrated for two values of the imperfect parameters $K=1,10$. The effective properties in the composite augments as the spring factor $K$ increases as well. The configuration of the cells affects the anisotropic properties of the composite. For example, composites with periodic cells $\theta=45^{\circ}, 75^{\circ}$ have monoclinic symmetry which is different to composites with configuration of hexagonal $\left(\theta=60^{\circ}\right)$ and square $\left(\theta=90^{\circ}\right)$ cells where the symmetry groups are $6 \mathrm{~mm}$ and $4 \mathrm{~mm}$ respectively.

\subsection{Effect of the imperfect adherence in the ultrasonic transducers applications}

Various parameters have been used to evaluate the performance of 1-3 piezoelectric composites. For underwater acoustic transducer or hydrophone, the measure of performance studied here are the hydrostatic charge coefficient $d_{h}$, hydrostatic voltage coefficient $g_{h}$, and hydrophone figure of merit $d_{h} g_{h}$. All these parameters are referred to 1-3 piezoelectric composites and therefore the upper symbol "*" means overall properties of the composite but for simplicity it is omitted in the parameters. For instance, the hydrostatic charge coefficient $d_{h}$ is defined by

$d_{h}=d_{13}^{*}+d_{23}^{*}+d_{33}^{*}$.

The corresponding hydrostatic voltage coefficient $g_{h}$ is defined by

$g_{h}=\frac{\left(d_{h}^{*}\right)^{2}}{\kappa_{33}^{*}}$

A useful figure of merit is the product of $d_{h}$ and $g_{h}$. High values for these parameters indicate a high sensitivity of the transducer as it is explained by Avellaneda and Swart [18] and Gibiansky and Torquato [19].

Table 4

Behavior of the composite with perfect and imperfect contacts for hexagonal and square cells.

\begin{tabular}{lrrrrr}
\hline \multirow{2}{*}{$K_{n}=K_{t}=K_{s} \equiv K$} & \multicolumn{2}{l}{$60^{\circ}$} & & \multicolumn{2}{l}{$90^{\circ}$} \\
\cline { 2 - 3 } \cline { 5 - 6 } & \multicolumn{1}{l}{$K=5$} & $K=10^{12}$ & & \multicolumn{1}{l}{$K=5$} & $K=10^{12}$ \\
\hline$C_{11}^{*}(\mathrm{GPa})$ & 104.07 & 154.24 & 104.30 & 154.32 \\
$C_{12}^{*}(\mathrm{GPa})$ & 40.83 & 78.73 & & 40.09 & 78.65 \\
$C_{13}^{*}(\mathrm{GPa})$ & 46.26 & 74.75 & & 46.12 & 74.75 \\
$C_{33}^{*}(\mathrm{GPa})$ & 114.83 & 133.26 & & 114.75 & 133.26 \\
$C_{44}^{*}(\mathrm{GPa})$ & 32.21 & 34.66 & & 32.18 & 34.65 \\
$C_{66}^{*}(\mathrm{GPa})$ & 31.62 & 37.75 & & 31.23 & 37.67 \\
$e_{15}^{*}\left(\mathrm{C} / \mathrm{m}^{2}\right)$ & 10.16 & 11.01 & & 10.17 & 11.01 \\
$e_{31}^{*}\left(\mathrm{C} / \mathrm{m}^{2}\right)$ & -2.06 & -3.15 & & -2.04 & -3.15 \\
$e_{33}^{*}\left(\mathrm{C} / \mathrm{m}^{2}\right)$ & 13.32 & 12.61 & & 13.32 & 12.61 \\
$\kappa_{11}^{*}(\mathrm{nC} / \mathrm{Vm})$ & 10.17 & 9.88 & & 10.16 & 9.87 \\
$\kappa_{33}^{*}(\mathrm{nC} / \mathrm{Vm})$ & 8.25 & 8.30 & 8.25 & 8.30 \\
\hline
\end{tabular}

Table 3

Comparison between AHM and FEM for 1-3 piezocomposite system made of $\mathrm{BaTiO}_{3}$ matrix and PZT-7A fiber.

\begin{tabular}{|c|c|c|c|c|c|c|c|c|c|c|c|}
\hline & $C_{11}^{*}(\mathrm{GPa})$ & $C_{12}^{*}(\mathrm{GPa})$ & $C_{13}^{*}(\mathrm{GPa})$ & $C_{33}^{*}(\mathrm{GPa})$ & $C_{44}^{*}(\mathrm{GPa})$ & $C_{66}^{*}(\mathrm{GPa})$ & $e_{15}^{*}\left(\mathrm{C} / \mathrm{m}^{2}\right)$ & $e_{31}^{*}\left(\mathrm{C} / \mathrm{m}^{2}\right)$ & $e_{33}^{*}\left(\mathrm{C} / \mathrm{m}^{2}\right)$ & $\kappa_{11}^{*}(\mathrm{nC} / \mathrm{Vm})$ & $\kappa_{33}^{*}(\mathrm{nC} / \mathrm{Vm})$ \\
\hline AHM & 155.1 & 80.9 & 76.3 & 131.3 & 33.3 & 37.0 & 10.9 & -3.0 & 11.8 & 9.4 & 7.2 \\
\hline FEM & 155.0 & 81.0 & 76.3 & 131.3 & 33.3 & 37.1 & 10.9 & -2.9 & 11.8 & 9.4 & 7.2 \\
\hline
\end{tabular}


For biomedical imaging applications, the piezoelectric electromechanical coupling $k_{t}$ and the acoustic impedance $Z$ are used to measure the performance of the medical ultrasonic imaging transducers, where low acoustic impedance and high electromechanical coupling is desired. See more details in the works of Smith et al. [29] and Smith and Auld [30]. The electromechanical coupling $k_{t}$ is defined as

$k_{t}=\sqrt{1-\frac{C_{33}^{* E}}{C_{33}^{* D}}}$

where

$C_{33}^{* D}=C_{33}^{* E}+\frac{e_{33}^{* 2}}{\kappa_{33}^{*}}$.

The acoustic impedance $Z$ is given by

$Z=\left(C_{33}^{* D} \rho\right)^{1 / 2}$

where the composite density $\rho$ is determined

$\rho=V_{2} \rho_{2}+\left(1-V_{2}\right) \rho_{1}$

and where $\rho_{2}$ is the density of the fiber and $\rho_{1}$ is the density of the matrix.

In Figs. 3-6 the configuration of the periodic cell is hexagonal. Figs. 3 and 4 show the effective hydrostatic charge coefficient $d_{h}$ and the figure of merit $d_{h} g_{h}$ of the piezoelectric composite with respect to the volume fraction of the PZT-7A fibers for Araldite D matrix material. Three different values of the normal imperfection parameters $\left(K_{n}=1,10,10^{12}\right)$ are used in the computation where the tangential imperfect parameter taken $K_{t}=K_{s}=10^{12}$. It can be seen that the curve $K_{n}=1$ is superior to the other two curves. We could suppose that the consideration of imperfect adherence is not good for the performance of the composites. However, sometimes the imperfection at the interface provokes certain improvements in some properties of the composites for different applications. This fact could be considered as a paradoxical behavior, for example, the normal imperfection improves the properties $d_{h}, d_{h} g_{h}$ and $k_{t}$ of the composite (Figs. 3-5). Low values of mechanical imperfection parameters induce a weaker bonded at the contact between matrix and fiber in the composite. In this case the normal parameter imperfection for low adherence between fiber and matrix improves the property $d_{h}$ and $d_{h} g_{h}$ of the 1-3 piezoelectric composites, and it can be seen that the $d_{h}$ and $d_{h} g_{h}$ for low values of $K_{n}$ exceed the value of the composite with perfect contact. The maximum value for the hydrostatic figure of merit $d_{h} g_{h}$ increases with decreasing the normal parameter of imperfection (Fig. 4). This maximum value is achieved at a low volume fraction of the piezoceramic fibers $\left(V_{2}<15 \%\right)$.

For purpose of verifying the validity of the method used in the present study, the calculated electromechanical coupling effective factor $k_{t}$ is compared in Fig. 5 with existing experimental results of Chan and Unsworth [31] for perfect contact. The material properties in Table 2 and three different values of the normal imperfection parameters $\left(K_{n}=1,10,10^{12}\right)$ and the tangential imperfect parameter $K_{t}=K_{s}=10^{12}$ were used for the calculations. The electromechanical coupling effective coefficient $k_{t}$ characterizes the transformation of electric to mechanical energy and conversely in the $x_{3}$ axial direction. High electromechanical coupling factor is important for designing pulse-echo ultrasonic transducer application. This parameter $k_{t}$ for contact perfect is superior to the conventional piezoelectric material PZT-7A in almost all the range of fiber volume fraction. When the normal imperfect parameter $K_{n}$ diminishes then the conversion between electrical and mechanical energy diminishes in the normal direction as well and by conservation of energy there exist an augment of conversion of energy in the axial direction and it is the reason of the increment of $k_{t}$. In Fig. 5, the data points of the experiment are scattered around the predictions with different imperfect parameters which represent different bonding condition for the PZT-7A-Araldite $\mathrm{D}$ composite. Notice that the curves related to imperfect parameters have the same trend. Particularly the curve $K_{n}=1$ is higher than

Table 5

Comparison between AHM with spring parameter $K=10^{12}$ and EEVM, for perfect contact and four different fiber arrays.

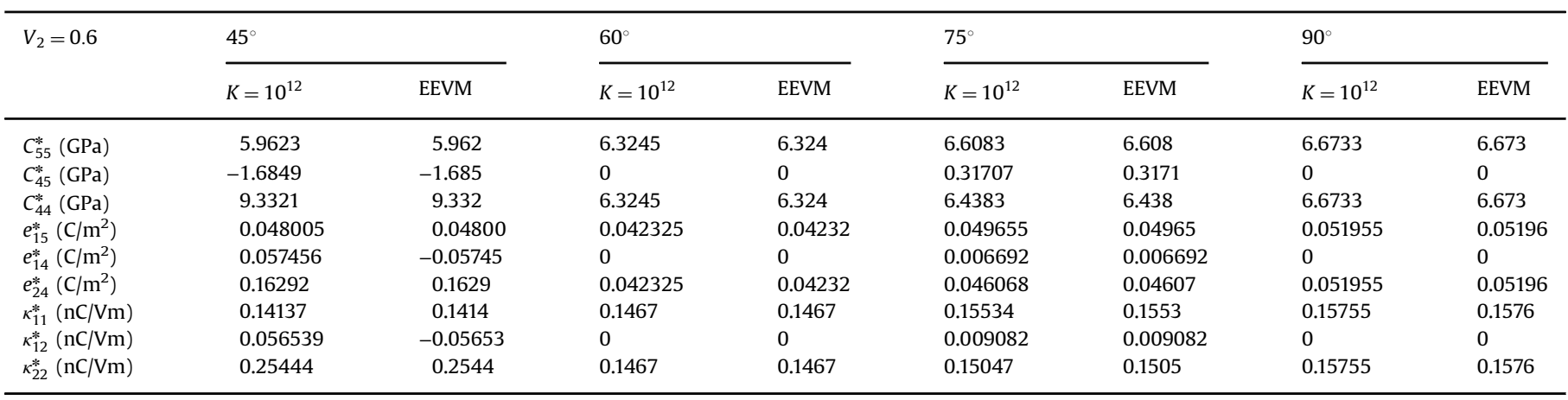

Table 6

Influence of the imperfect spring parameter $K$ in the global behavior of the composite.

\begin{tabular}{|c|c|c|c|c|c|c|c|c|}
\hline \multirow[t]{2}{*}{$V_{2}=0.6$} & \multicolumn{2}{|l|}{$45^{\circ}$} & \multicolumn{2}{|l|}{$60^{\circ}$} & \multicolumn{2}{|l|}{$75^{\circ}$} & \multicolumn{2}{|l|}{$90^{\circ}$} \\
\hline & $K=1$ & $K=10$ & $K=1$ & $K=10$ & $K=1$ & $K=10$ & $K=1$ & $K=10$ \\
\hline$C_{55}^{*}(\mathrm{GPa})$ & 1.7593 & 4.495 & 1.7594 & 4.795 & 1.7593 & 4.8786 & 1.7593 & 4.8731 \\
\hline$C_{45}^{*}(\mathrm{GPa})$ & 0.0003 & -0.5483 & 0 & 0 & $-4.18 \times 10^{-5}$ & 0.13935 & 0 & 0 \\
\hline$C_{44}^{*}(\mathrm{GPa})$ & 1.7587 & 5.5916 & 1.7594 & 4.795 & 1.7594 & 4.804 & 1.7593 & 4.8731 \\
\hline$e_{15}^{*}\left(\mathrm{C} / \mathrm{m}^{2}\right)$ & 0.009 & 0.0306 & 0.00940 & 0.03120 & 0.010134 & 0.035193 & 0.010328 & 0.036115 \\
\hline$e_{14}^{*}\left(\mathrm{C} / \mathrm{m}^{2}\right)$ & -0.0049 & -0.0277 & 0 & 0 & 0.000777 & 0.004136 & 0 & 0 \\
\hline$e_{24}^{*}\left(\mathrm{C} / \mathrm{m}^{2}\right)$ & 0.0188 & 0.086 & 0.0094 & 0.0312 & 0.009717 & 0.032976 & 0.010328 & 0.036115 \\
\hline$\kappa_{11}^{*}(\mathrm{nC} / \mathrm{Vm})$ & 0.142 & 0.1417 & 0.1469 & 0.1468 & 0.15567 & 0.15546 & 0.15792 & 0.1577 \\
\hline$\kappa_{12}^{*}(\mathrm{nC} / \mathrm{Vm})$ & -0.0577 & -0.0572 & 0 & 0 & 0.009155 & 0.009112 & 0 & 0 \\
\hline$\kappa_{22}^{*}(\mathrm{nC} / \mathrm{Vm})$ & 0.2574 & 0.2561 & 0.1469 & 0.1468 & 0.15076 & 0.15058 & 0.15792 & 0.1577 \\
\hline
\end{tabular}




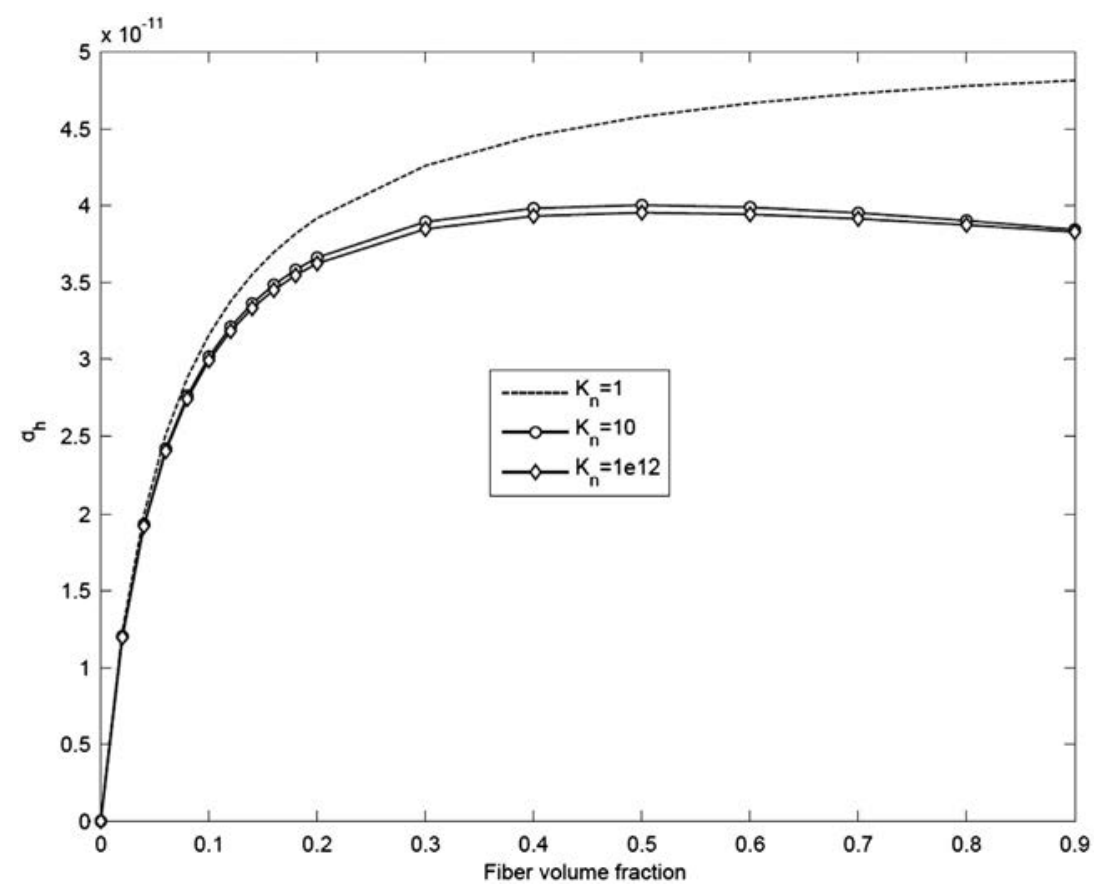

Fig. 3. Effective hydrostatic charge coefficient $d_{h}$ as a function of fiber volume fraction. Various normal imperfect parameters for 1-3 piezoelectric composites.

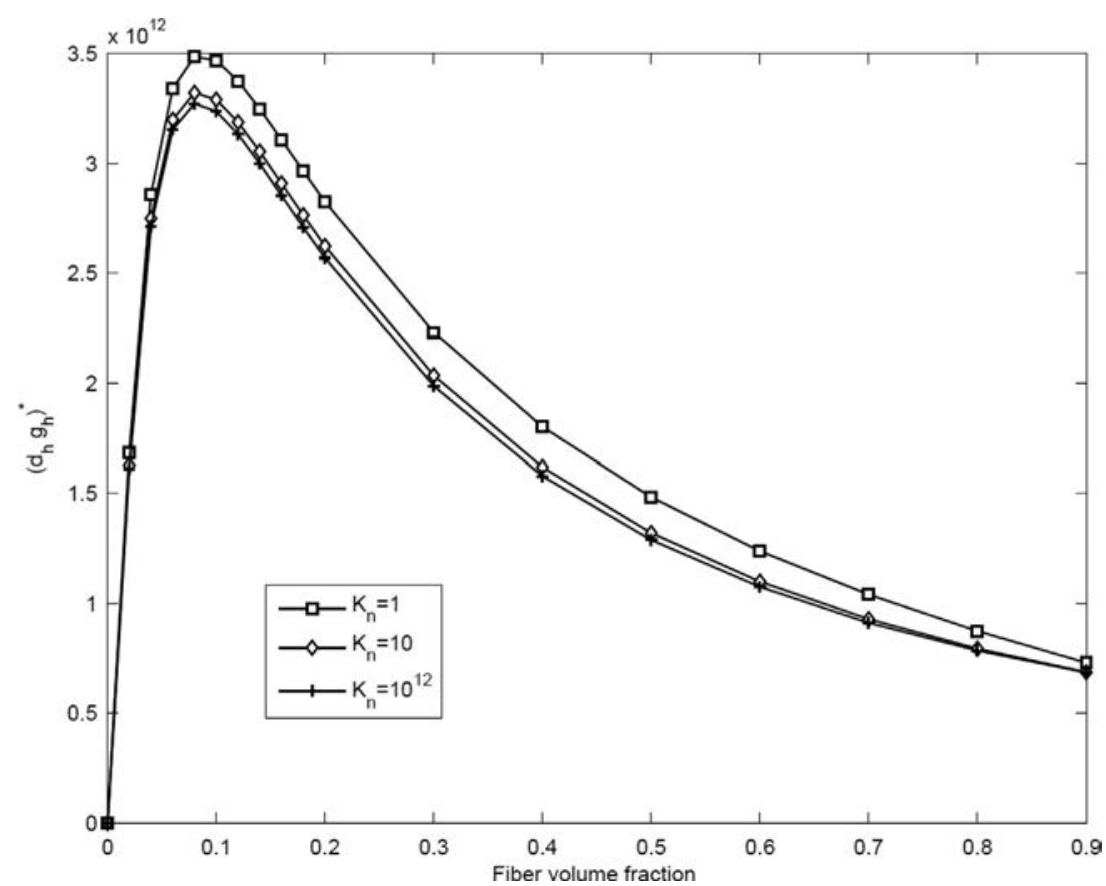

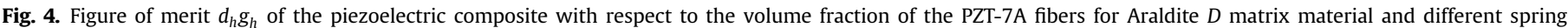
parameters.

the remaining curve which is desired for biomedical imaging applications. $k_{t}$ increases as the PZT-7A volume fraction increases. The increase becomes significant for a volume fraction greater than $50 \%$.

In Fig. 6, the effective electromechanical coupling $k_{t}$ versus the effective acoustic impedance $Z$ for the piezoelectric composite with three different values of the normal imperfection parameters $\left(K_{n}=1,10,10^{12}\right)$ is shown where the tangential imperfect parameter is taken $K_{t}=10^{12}$. It can be seen the influence of the imperfect interface in the behavior of this composite, which is useful for the design of medical imaging transducers. It can be seen that $k_{t}$ first increases rapidly with $Z$ and notice that the curves for imperfect cases
$K_{n}=1, \quad 10$ are higher than the curve of perfect contact $K_{n}=10^{12}$ where a slight diminish is achieved for high fiber volume fraction. $k_{t}$ further increases as the normal imperfect parameter decreases with the same value of $Z$. The conversion between electrical and mechanical energy diminishes in the normal direction as $K_{n}$ diminishes then there exits an increment of $k_{t}$ for a fixed value of the impedance or fixed value of the fiber volume fraction.

Some limitations of the obtained solutions would be mentioned. The determination of the effective properties required the convergence of the series (31) and (41). These series expansion depend on the contrast between the properties of the components and on the volume fraction of the fibres. In the case of a high-contrast composite 


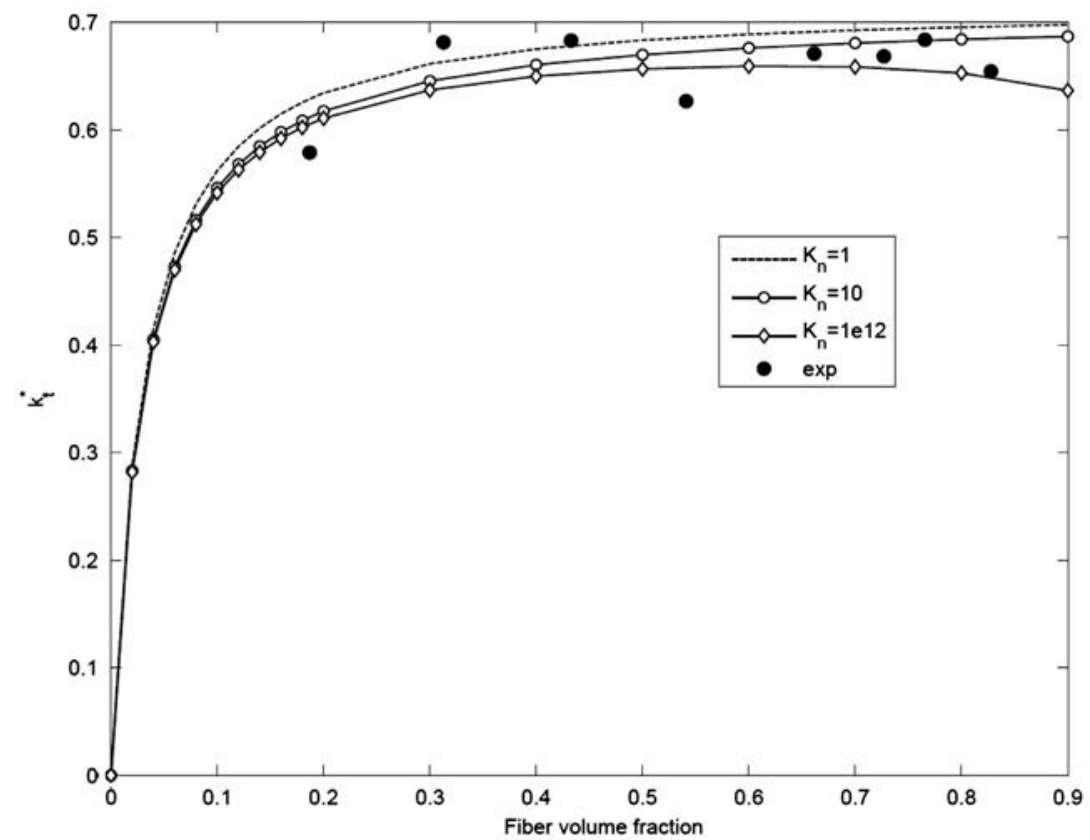

Fig. 5. Comparison of the calculated electromechanical coupling $k_{t}$ with the experimental data of Chan and Unsworth [31].

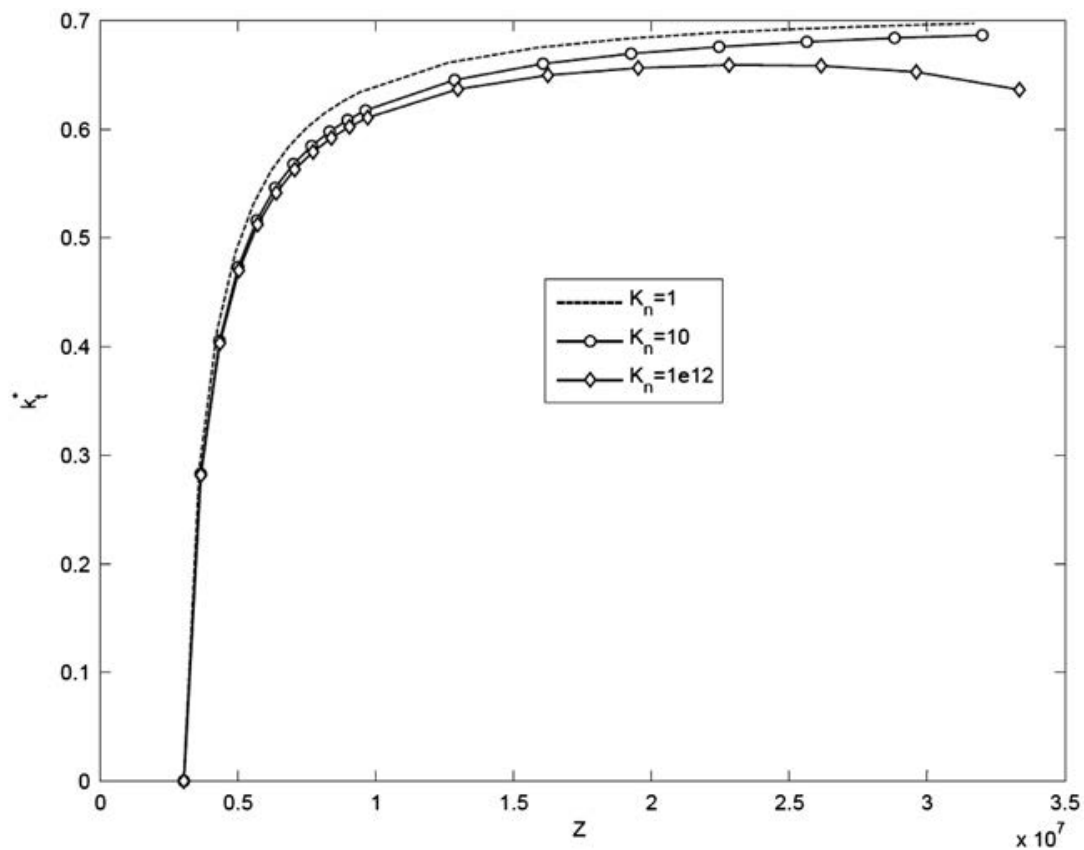

Fig. 6. $k_{t}$ vs. $Z$ for 1-3 piezoelectric composites with PZT-7A fibers and various value of imperfection parameter.

with densely-packed fibres, the gradients of the local fields can grow significantly. Then, the convergence of the series decreases and evaluation of the accurate numerical results may become very timeconsuming. In this sense, the above computations were made for $N_{0}=10$, where $N_{0}$ denotes the number of equations considered in the solution of the infinite algebraic system of Eqs. (34) and (48). The solution to the infinite order algebraic system (34) and (48) is achieved by means of truncation to an infinite order and the Cramer's rule. A fast convergence of successive truncations is ensured because the system is regular (see references in Bravo-Castillero et al. (2001)) so that successive approximations can be applied. In general, for low volume fraction of fiber $\left(V_{2}<0.4\right)$ the accuracy and convergence of the results are good for much smaller values of $N_{0}\left(N_{0} \leq 2\right)$. More terms are required for high volume fraction of fibers as well as high contrast of fiber and matrix, in particular, $N_{0}>10$ gives an approximation with absolute error less than $1 \%$. The absolute error between two consecutive truncations is very low.

The explicit analytical form of the effective coefficients (37), (50)-(64) are large and complicated. They require computation of a number of terms of series (31) and (41) and the solution of the system (34) and (48). However, once the computational program is established the time-consuming is very short.

\section{Conclusion}

In the present paper, an asymptotic approach for simulation of the imperfect interfacial bonding in composite materials is proposed by means of spring model. In the asymptotic limit, we can simulate different degrees of the interface's response: the case $\mathbf{K} \rightarrow \infty$ 
corresponds to the perfect bonding, the case $\mathbf{K} \rightarrow 0$ to the complete separation of the matrix and inclusions. As illustrative examples we consider fibre-reinforced composites with different angular configurations of unit cells. The analysis is based on the asymptotic homogenization method, the cell problem is solved using the theory of complex variable. The local problems associated to an anisotropic piezoelectric composite with mechanical imperfect interface condition and parallelogram cell are formulated and solved. As the results we obtain approximate analytical solutions for the effective moduli on micro level depending on the degree of the interfacial debonding. Developed solutions are valid for all values of the components' volume fractions and properties. The effect of the inclination of the cell affects the anisotropic character of the composites and in general this effect leads to different crystalline symmetries in the global behavior of the composite, for instance, tetragonal $4 \mathrm{~mm}$ class symmetry (6 elastic, 3 piezoelectric and 2 dielectric permittivity effective moduli) for square periodic cell; hexagonal $6 \mathrm{~mm}$ class symmetry ( 5 elastic, 3 piezoelectric and 2 dielectric permittivity effective moduli) for hexagonal periodic cell; orthorhombic class symmetry ( 9 elastic, 3 piezoelectric and 3 dielectric permittivity effective moduli) for rectangular periodic cell and finally monoclinic type 2 (13 elastic, 8 piezoelectric and 4 dielectric permittivity effective moduli) anisotropic behavior in the composite with parallelogram cell. On the other hand, it follows from the analysis of the results that in the case of the perfect electric bonding, softening the rigidity of the interface does have an influence on the effective elastic moduli, but has a very minor effect on the electric properties of the material.

A parametric study has been conducted to study the influence of the imperfect contact on the electro-mechanical properties on the performance of the 1-3 piezoelectric composite for the designing of transducer applications. Based on the study, we can say

1. The presence of normal imperfect contact enhances the effective hydrostatic performances of the 1-3 piezoelectric composites. The peak values of the performance parameter in the figure of merit increases as the normal imperfect contact diminishes.

2. The normal imperfection contact improves the performance of the 1-3 piezoelectric composite by increasing the electromechanical coupling while reducing the acoustic impedance of the composite. This performance is improved as $K_{n}$ diminishes.

\section{Acknowledgments}

The funding of conacyt project number 129658 is gratefully acknowledged. The authors gratefully acknowledge to the project SHICHAN supported by FSP (Cooperation Scientifique FrancoCubaine). This work was partially written while R. RodríguezRamos was visiting the Laboratoire de Mécanique et d'Acoustique (LMA-CNRS), Marseille, France. Thanks to Ecole Central of Marseille for the support given for the visit. Thanks to the reviewers for the valuable comments and suggestions and to Ramiro Chávez Tovar and Ana Pérez Arteaga for computational assistance.

\section{References}

[1] Hashin Z. Thermoelastic properties of fiber composites with imperfect interface. Mech Mater 1990;8:333-48.

[2] Bigoni D, Serkov SK, Valentini M, Movchan AB. Asymptotic models of dilute composites with imperfectly bonded inclusions. Int J Solids Struct 1998;35:3239-58
[3] Andrianov IV, Bolshakov VI, Danishevs'kyy VV, Weichert D. Asymptotic simulation of imperfect bonding in periodic fibre-reinforced composite materials under axial shear. Int J Mech Sci 2007;49:1344-54.

[4] Yanase K, Ju JW. Effective elastic moduli of spherical particle reinforced composites containing imperfect interfaces. Int J Damage Mech 2012;21:97-127.

[5] Chen X, Ye J, Zhai Z, He Z. Micromechanical analysis of off-axis loading of fiber reinforced composites with imperfect interface bonding. Int J Mech Sci 2012:54:113-20.

[6] Shu X. Piezothermoelastic responses of piezoelectric composite laminates with weak interfaces. Acta Mech 2010;214:327-40.

[7] Shodja HM, Tabatabaei SM, Kamali MT. A piezoelectric-inhomogeneity system with imperfect interface. Int J Eng Sci 2006;44:291-311.

[8] Andrianov IV, Bolshakov VI, Danishevs'kyy VV, Weichert D. Asymptotic study of imperfect interfaces in conduction through a granular composite material. Proc R Soc London A 2010;466:2707-25.

[9] Shodja HM, Tabatabaei SM, Kamali MT. A piezoelectric medium containing a cylindrical inhomogeneity: Role of electric capacitors and mechanical imperfections. Int J Solids Struct 2007;44:6361-81.

[10] Bravo-Castillero J, Guinovart Díaz R, Sabina FJ, Rodríguez Ramos R. Closedform expressions for the effective coefficients of a fiber-reinforced composite with transversely isotropic constituents-II. Piezoelectric and square symmetry. Mech Mater 2001;33(4):237-48.

[11] Sabina FJ, Rodríguez Ramos R, Bravo Castillero J, Guinovart Díaz R. Closed-form expressions for the effective coefficients of fibre-reinforced composite with transversely isotropic constituents-II: piezoelectric and hexagonal symmetry. J Mech Phys Solids 2001;49:1463-79.

[12] Guinovart-Díaz R, López-Realpozo JC, Rodríguez-Ramos R, Bravo-Castillero J, Ramírez M, Camacho-Montes $\mathrm{H}$, et al. Influence of parallelogram cells in the axial behaviour of fibrous composite. Int J Eng Sci 2011;49 75-84.

[13] Guinovart-Díaz R, Yan P, Rodríguez-Ramos R, López-Realpozo JC, Jiang CP, Bravo-Castillero J, et al. Effective properties of piezoelectric composites with parallelogram periodic cells. Int J Eng Sci 2012;53 58-66.

[14] Rodríguez-Ramos R, Yan P, López-Realpozo JC, Guinovart-Díaz R, Bravo-Castillero J, Sabina FJ, et al. Two analytical models for the study of periodic fibrous elastic composite with different unit cells. Comp Struct 2011;93:709-14.

[15] Molkov VA, Pobedria BE. Effective elastic properties of a composite with elastic contact. Izvestia Akademia Nauk SSR, Mekh Tverdovo Tela 1988;1:111-7.

[16] Rodríguez-Ramos R, Guinovart-Diaz R, López JC, Bravo-Castillero J, Sabina FJ. Influence of imperfect elastic contact condition on the antiplane effective properties of piezoelectric fibrous composites. Arch Appl Mech 2010;80:377-88.

[17] Lopez-Realpozo JC, Rodriguez-Ramos R, Guinovart-Diaz R, Bravo-Castillero J, Sabina FJ. Transport properties in fibrous elastic rhombic composite with imperfect contact condition. Int J Mech Sci 2011;53:98-107.

[18] Avellaneda M, Swart PJ. Calculating the performance of 1-3 piezoelectric composites for hydrophone applications: an effective medium approach. J Acoust Soc Am 1998;103:1449-67.

[19] Gibiansky LV, Torquato S. On the use of homogenization theory to design optimal piezocomposites for hydrophone applications. J Mech Phys Solid 1997;45:689-708.

[20] Bakhvalov NS, Panasenko GP. Homogenization averaging processes in periodic media. Dordrecht: Kluwer; 1989.

[21] Bensoussan A, Lions JL, Papanicolaou G. Asymptotic analysis for periodic structures. New York: North-Holland; 1978.

[22] Pobedria BE. Mechanics of composite materials. Moscow: Moscow State University Press; 1984 (in Russian).

[23] Royer D, Dieulesaint E. Elastic waves in solids I. Berlin, Heidelberg: Springer Verlag; 2000.

[24] Chih-Bing L. Tables of values of $\sigma_{2 s}$ relating to Weierstrass' elliptic function. Math Comput 1965;19(89):123-7.

[25] Rodriguez-Ramos R, Sabina FJ, Guinovart-Díaz R, Bravo-Castillero J. Closed form expressions for the effective coefficients of fibre-reinforced composite with transversely isotropic constituents - I. Elastic and square symmetry. Mech Mat 2001;33:223-35.

[26] Smith WA, Shaulov AA, Auld BA. Tailoring the properties of composite piezoelectric materials. In: Proceedings of the IEEE ultrasonics symposium; 1985. pp. 642-47.

[27] Smith WA, Auld BA. Modeling 1-3 composite piezoelectrics: thickness mode oscillations. IEEE Trans Ultrason Ferroelectr Freq Control 1991;38:40-7.

[28] Kar-Gupta R, Venkatesh TA. Electromechanical response of 1-3 piezoelectric composites: Effect of poling characteristics. J Appl Phys 2005;98:054102.

[29] Levin VM, Rakovskaja MI, Kreher WS. The effective thermoelectroelastic properties of microinhomogeneous materials. Int J Solids Struct 1999;36:2683-705.

[30] Yan P, Jiang CP, Song F. An eigenfunction expansion-variational method for the anti-plane electroelastic behavior of three-phase fiber composites. Mech Mat 2011;43:586-97.

[31] Chan HLW, Unsworth J. Simple model for piezoelectric ceramic/polymer 1-3 composites used in ultrasonic transducer applications. IEEE Trans Ultrason Ferroelectr Freq Control 1989;36:434-41. 